\title{
Direct Cosmological Simulations of the Growth of Black Holes and Galaxies
}

\section{Citation}

Di Matteo, Tiziana, Jörg Colberg, Volker Springel, Lars Hernquist, and Debora Sijacki. 2008. "Direct Cosmological Simulations of the Growth of Black Holes and Galaxies." The Astrophysical Journal 676 (1): 33-53. https://doi.org/10.1086/524921.

\section{Permanent link}

http://nrs.harvard.edu/urn-3:HUL.InstRepos:41381810

\section{Terms of Use}

This article was downloaded from Harvard University's DASH repository, and is made available under the terms and conditions applicable to Other Posted Material, as set forth at http:// nrs.harvard.edu/urn-3:HUL.InstRepos:dash.current.terms-of-use\#LAA

\section{Share Your Story}

The Harvard community has made this article openly available.

Please share how this access benefits you. Submit a story.

Accessibility 
SubmitTed to ApJ 05/14/07

Preprint typeset using $\mathrm{LT}_{\mathrm{E}} \mathrm{X}$ style emulateapj v. 6/22/04

\title{
DIRECT COSMOLOGICAL SIMULATIONS OF THE GROWTH OF BLACK HOLES AND GALAXIES
}

\author{
Tiziana Di Matteo, ${ }^{1}$ Jörg Colberg,,${ }^{1}$ Volker Springel, ${ }^{2}$ Lars Hernquist ${ }^{3}$ \& Debora SiJaCKI ${ }^{2}$ \\ Submitted to ApJ 05/14/07
}

\begin{abstract}
We investigate the coupled formation and evolution of galaxies and their embedded supermassive black holes using state-of-the-art hydrodynamic simulations of cosmological structure formation. For the first time, we selfconsistently follow the dark matter dynamics, radiative gas cooling, star formation, as well as black hole growth and associated energy feedback processes, starting directly from initial conditions appropriate for the $\Lambda \mathrm{CDM}$ cosmology. Our modeling of the black hole physics is based on an approach we have recently developed and tested in simulations of isolated galaxy mergers. Here we apply the same model in cosmological simulations to examine: (i) the predicted global history of black hole mass assembly in galaxies, (ii) the evolution of the local black hole-host mass correlations and (iii) the conditions that allow rapid growth of the first quasars, as well as the properties of their hosts and descendants today. We find that our simulations produce a total black hole mass density $\rho_{\mathrm{BH}} \simeq 2 \times 10^{5} \mathrm{M}_{\odot} \mathrm{Mpc}^{-3}$ by $z=0$, in good agreement with observational estimates. The black hole accretion rate density, $\dot{\rho}_{\mathrm{BH}}$, peaks at lower redshift and evolves more strongly at high redshift than the star formation rate density, $\dot{\rho}_{*}$, with an approximate scaling as $\dot{\rho}_{\mathrm{BH}} / \dot{\rho}_{*} \propto(1+z)^{-4}$ at $z \geq 3$. On the other hand, the ratio $\rho_{\mathrm{BH}} / \rho_{*} \sim(1+z)^{-0.6}$ of black hole to stellar mass densities shows only a moderate evolution at low redshifts $z \lesssim 3$. For the population of galaxies identified in the simulations at $z=1$ we find strong correlations between black hole mass and velocity dispersion or mass of the stellar systems. The predicted correlations agree well with the measured local $M_{\mathrm{BH}}-\sigma$ and $M_{\mathrm{BH}}-M_{*}$ relationships, but also suggest a weak evolution with redshift in the normalization, and in particular the slope. However, the magnitude of this effect is sensitive to the range of masses being probed. For stellar masses of $M_{*} \geq 3 \times 10^{10}$, we predict a trend of increasing $M_{*} / M_{\mathrm{BH}}$ with redshift, in agreement with recent direct estimates of the $\mathrm{BH}$ to host stellar mass ratio at high redshift and the conjecture that a more fundamental relation (a BH fundamental plane) should involve both $M_{*}$ and $\sigma$. We find that our simulation models can also produce quite massive black holes at high redshift, as a result of extended periods of exponential growth in relatively isolated, rare regions that collapse early and exhibit strong gas inflows. Interestingly, when followed to their descendants, these first supermassive BH systems are not necessarily the most massive ones today, since they are often overtaken in growth by quasars that form later.
\end{abstract}

Subject headings: quasars: general — galaxies: formation — galaxies: active — galaxies: evolution — cosmology: theory - hydrodynamics

\section{INTRODUCTION}

Following the discovery of quasars (Schmidt 1963; Greenstein \& Matthews 1963) it was suggested that supermassive black holes $\left(10^{6}-10^{9} \mathrm{M}_{\odot}\right)$ lie at the centers of galaxies, and that the quasar activity is fueled by the release of gravitational energy from their accreted matter. The remnants of quasar phases at early times are probably the supermassive black holes found at the centers of galaxies in our local Universe. Interestingly, the properties of these supermassive black holes are tightly coupled to the mass (Magorrian et al. 1998) and velocity dispersion of their host galaxies, as manifested in the $M_{\mathrm{BH}}-\sigma$ relation of spheroids (Ferrarese \& Merritt 2000; Gebhardt et al. 2000). In addition, the black hole mass is correlated with the concentration or Sersic index (Graham \& Driver 2006). Most recently, Hopkins et al. (2007a) have shown that these various correlations are not independent, and can be understood as projections of a "black hole fundamental plane" (BHFP), similar to that describing properties of elliptical galaxies.

\footnotetext{
${ }^{1}$ Physics Department, Carnegie Mellon University, 5000 Forbes Avenue, Pittsburgh, PA 15213

2 Max-Planck-Institut für Astrophysik, Karl-Schwarzchild-Straße 1, 85740 Garching bei München, Germany

${ }^{3}$ Harvard-Smithsonian Center for Astrophysics, 60 Garden Street, Cambridge, MA 02138, USA
}

The existence of highly luminous quasars also constrains the formation and evolution of massive galaxies and the epoch of reionization. Quasars with inferred black hole masses in excess of $10^{9} \mathrm{M}_{\odot}$ have now been discovered out to $z \sim 6$ (Fan et al. 2003), indicating an early formation time for black holes and galaxy spheroids and posing a significant challenge for theoretical models of high-redshift quasar and galaxy formation.

This growing observational evidence, drawn from local galaxies to high redshift quasars, argues for a close connection between the formation and evolution of galaxies and of their central supermassive black holes. However, the physical nature of this relationship has yet to be understood in detail. Indeed, there are significant gaps in our observational and theoretical knowledge of the history of black hole formation and evolution in galaxies.

For example, current velocity dispersion measurements are inconclusive about the important question whether the tight scaling relations evolve with redshift (Woo et al. 2006; Shields et al. 2006), or are essentially invariant (Shields et al. 2003) as a function of time. We note that some evolution in the ratio of black hole to halo mass is suggested by clustering constraints (e.g Adelberger \& Steidel 2005; Lidz et al. 2006), but these measurements do not directly address the relationship between black hole and properties of the luminous host galaxy. More relevant are comparisons of the black 
hole mass inferred from quasar observations to the host stellar mass, both observationally (Merloni 2004) and theoretically (Hopkins et al. 2006a), which indicate an evolution in e.g. the Magorrian relation, in the sense that black holes are more massive relative to luminous spheroids at high redshifts than at $z=0$.

Theoretical studies of the co-evolution of black holes and galaxies have so far mostly used so-called semianalytical modeling (e.g. Kauffmann \& Haehnelt 2000; Cattaneo et al. 1999; Wvithe \& Loeb 2003; Volonteri et al. 2003; Di Matteo et al. 2003; Granato et al. 2004; Springel et al. 2005c; Cattaneo et al. 2005; Croton et al. 2006; De Lucia et al. 2006; Malbon et al. 2007) of galaxy formation, in which the growth of galaxies and their embedded black holes is followed with simple physical parameterizations on top of dark matter merging history trees. Many of these models assume that quasar activity is triggered by major galaxy mergers, motivated by hydrodynamical simulations that have shown that gravitational tidal fields during major mergers of gas rich galaxies produce strong gas inflows (Barnes \& Hernquist 1991, 1996), which lead to a burst of nuclear star formation (Mihos \& Hernquist 1996) and are likely the prerequisite for rapid black hole growth and quasar activity. Nearby quasars are indeed preferentially found in tidally disturbed objects (e.g Jogee 2004), corroborating the importance of galaxy interactions and mergers for major black hole growth.

Many theoretical explanations for the observed correlations between galaxy properties and black hole mass rely on some form of self-regulated growth of the BHs. For example, it has been suggested that the central black holes grow until they release sufficient energy to unbind the gas that feeds them from their host galaxy (Ciotti \& Ostriker 1997; Silk \& Rees 1998; Fabian 1999; Wyithe \& Loeb 2003). We recently explored such a local energy feedback for the first time with selfconsistent, fully three-dimensional hydrodynamic simulations of galaxies (Di Matteo et al. 2005; Springel et al. 2005b) that include a treatment of accretion on supermassive black holes and their associated energy feedback. These simulations have demonstrated that the fundamental $\mathrm{BH}$-host correlation including the $M_{\mathrm{BH}}-\sigma$ relation can indeed be reproduced in feedback-regulated models of $\mathrm{BH}$ growth (Di Matteo et al. 2005), in accordance with theoretical conjectures. At the same time, the dynamical coupling in the simulations of hydrodynamical gas inflow, star formation, black hole growth and associated feedback processes gives them substantial predictive power well beyond that of simplified analytical and semi-analytical models. Besides the $M_{\mathrm{BH}}-\sigma$ or $M_{\mathrm{BH}}-M_{*}$ (Di Matteo et al. 2005; Robertson et al. 2006b) relationships, the simulation models can for example predict the detailed properties of the spheroidal galaxies forming in major mergers and how they correlate with the $\mathrm{BH}$ masses. In fact, they suggest the existence of a fundamental plane relation for BHs $\left(M_{B H} \propto \sigma^{3.0} R_{e}^{0.5}\right.$ (Hopkins et al. 2007a), provide an explanation for the red colors of massive elliptical galaxies (Springel et al. 2005a), and describe the fundamental plane of elliptical galaxies (Robertson et al. 2006a). They also suggest luminosity-dependent quasar lifetimes, leading to a new interpretation for the origin of the quasar luminosity function and its evolution over cosmic history (Hopkins et al. 2005, 2006a).

In the present paper, we extend these earlier studies by carrying out fully cosmological hydrodynamic simulations of the $\Lambda$ CDM model that jointly follow the growth of galax- ies and supermassive black holes, as well as their associated feedback processes. Our approach is based on the same methodology that we have developed and applied in the highresolution simulations of galaxy mergers, augmented with a suitable mechanism to seed emerging new dark matter halos with a small black hole that can then grow by gas accretion later on. While much more restricted in numerical resolution than simulations of individual galaxy mergers, our modeling of star formation and black hole physics in terms of a subresolution treatment provides quite accurate results already at comparatively coarse resolution, an important prerequisite for attempting to model these processes in cosmological simulations. Nevertheless, numerical resolution is clearly an important limitation of our cosmological results, an aspect that we will discuss in more detail where appropriate. With this caveat in mind, we would like to stress however that the unambiguous initial conditions of direct cosmological simulation make them in principle the most powerful and accurate tool for studying the interplay of galaxy formation and black hole growth. Our aim is therefore to examine how well our current model for treating $\mathrm{BH}$ physics in simulations does in present state-of-the-art hydrodynamical calculations of cosmic structure formation, and what we can learn from them to advance our theoretical understanding of the co-evolution of galaxies and supermassive black holes. In this study we shall focus on basic properties of the black hole population, like the evolution of the cosmic $\mathrm{BH}$ mass density, and the correlations between $\mathrm{BH}$ masses and host galaxy properties. In Sijacki et al. (2007) we also study an extension of our feedback model with a 'radio mode' that is active at low accretion rates and is distinct from the normal quasar activity, allowing us to study the formation of AGN in rich galaxy clusters at low redshifts, where it is likely to be important.

This paper is structured as follows. In $\$ 2$ we describe our simulation set and the numerical modeling adopted for the ISM, star formation and gas accretion onto black holes. In $\S 3$ we present our results for the evolution of the global black hole mass density and compare it to the cosmic history of star formation and the evolution of the stellar density. In $\$ 4$ we examine our results for the fundamental $\mathrm{BH} /$ host correlations measured from the simulation and for their evolution from high to low redshift. Finally, we summarize and discuss our findings in $\S 5$.

\section{METHODOLOGY}

\subsection{Numerical code}

In this study we focus on a $\Lambda \mathrm{CDM}$ cosmological model with parameters chosen according to the first year results from the Wilkinson Microwave Anisotropy Probe (WMAP1; Spergel et al. 2003), $\Omega_{0}=0.3, \Omega_{\Lambda}=0.7$, Hubble constant $H_{0}=100 h \mathrm{~km} \mathrm{~s}^{-1} \mathrm{Mpc}^{-1}$ with $h=0.7$ and a scale invariant primordial power spectrum with index $n=1$, with a normalization of the amplitude of fluctuations $\sigma_{8}=0.9$. ${ }^{4}$. We use a significantly extended version of the parallel cosmological TreePM-SPH code GADGET2 (Springel 2005) to evolve a realization of $\Lambda \mathrm{CDM}$ initial conditions from high to low redshift. The combination of a high-resolution gravitational solver with individual and adaptive timesteps allows

\footnotetext{
${ }^{4}$ The largest simulation presented here had already been started by the time the updated third year constraints have become available (WMAP3; Spergel et al. 2006). We comment on effects on the growth of the halo mass function owing to the lower amplitude of fluctuations, $\sigma_{8}$ implied by WMAP3 in Li et al. (2007); Siiacki et al. (2007)
} 
this code to bridge a large dynamic range both in length- and timescales. Gas dynamics is followed with the Lagrangian smoothed particle hydrodynamics (SPH) (e.g Monaghan 1992) technique, which we employ in a formulation that manifestly conserves energy and entropy, despite the use of fully adaptive SPH smoothing lengths (Springel \& Hernquist 2002). Radiative cooling and heating processes are computed as in Katz et al. (1996), with a spatially uniform photoionizing UV background that is imposed externally.

Within cosmological (or galaxy-sized) numerical simulations, it is presently (and for some time to come) not feasible to follow the physics of star formation and black hole accretion from first principles down to scales of individual stars or black holes. Any numerical model of galaxy formation therefore needs to make substantial approximations for some of the relevant physics on unresolved scales.

For modeling star formation and its associated supernova feedback we use the sub-resolution multiphase model for the interstellar medium developed by Springel \& Hernquist (2003a). In this model, a thermal instability is assumed to operate above a critical density threshold $\rho_{\text {th }}$, producing a two phase medium consisting of cold clouds embedded in a tenuous gas at pressure equilibrium. Stars form from the cold clouds, and short-lived stars supply an energy of $10^{51} \mathrm{ergs}$ to the surrounding gas as supernovae. This energy heats the diffuse phase of the ISM and evaporates cold clouds, thereby establishing a self-regulation cycle for star formation. $\rho_{\text {th }}$ is determined self-consistently in the model by requiring that the equation of state (EOS) is continuous at the onset of star formation. The cloud evaporation process and the cooling function of the gas then determine the temperatures and the mass fractions of the two hot and cold phases of the ISM, such that the EOS of the model can be directly computed as a function of density. The latter is encapsulating the self-regulated nature of star formation owing to supernovae feedback in a simple model for a multiphase ISM. As in the Springel \& Hernquist (2003a) model we have included a model for supernova-driven galactic winds with an initial wind speed of $v \sim 480 \mathrm{~km} \mathrm{~s}^{-1}$.

For the parameter settings adopted here, the model reproduces the observed star formation rate surface densities in isolated spiral galaxies (Kennicutt 1989, 1998). Using a large number of nested cosmological simulations, the approach we adopt (and the parameters we use) has also been shown to lead to a numerically converged estimate for the cosmic star formation history of the universe that agrees reasonably well with low redshift observations (Springel \& Hernquist 2003b; Hernquist \& Springel 2003). For the modeling of BH accretion and feedback we adopt a similar strategy as for star formation, which we discuss next.

\subsection{Accretion and Feedback from Supermassive black holes}

As for star formation, we adopt a sub-resolution model to capture the main features of accretion and associated feedback on supermassive black holes (as introduced in Springel et al. 2005b; Di Matteo et al.2005). To this end, we represent black holes by collisionless 'sink' particles that can grow in mass by accreting gas from their immediate environments, or by merging with other black holes. We estimate the gas accretion rate onto a black hole using a Bondi-Hoyle-Lyttleton parameterization (Bondi 1952; Bondi \& Hoyle 1944; Hoyle \& Lyttleton 1939). In this description, the accretion rate onto the black hole is given by $\dot{M}_{\mathrm{B}}=4 \pi\left[G^{2} M_{\mathrm{BH}}^{2} \rho\right] /\left(c_{s}^{2}+v^{2}\right)^{3 / 2}$ where $\rho$, $c_{s}$ are the density and sound speed of the ISM gas, respec- tively, and $v$ is the velocity of the black hole relative to the surrounding gas. In this study we also allow the black hole to accrete at mildly super-Eddington values and impose a maximum allowed accretion rate equal to $2 x$ Eddington rate, $\dot{M}_{\text {Edd }}$. As we shall see such conditions are generally achieved at high redshift. We note that the detailed relativistic accretion flow onto the black hole is unresolved in our simulations, but if the limitating factor for rapid growth of BHs lies in the largerscale gas distribution around the black hole, which is resolved, then the Bondi prescription should capture the dependence of the mean accretion rate onto the conditions of the gas in the region around the black hole.

The radiated luminosity, $L_{\mathrm{r}}$ is related to the gas accretion rate, $\dot{M}_{\mathrm{BH}}$, by the radiative efficiency $\eta=L_{\mathrm{r}} /\left(\dot{M}_{\mathrm{BH}} c^{2}\right)$, which simply gives the mass to energy conversion efficiency, set by the amount of energy that can be extracted from the innermost stable orbit of an accretion disk around a black hole. We will adopt a fixed value of $\epsilon_{\mathrm{r}}=0.1$, which is the mean value for a radiatively efficient (Shakura \& Sunyaev 1973) accretion disk onto a Schwarzschild black hole.

We assume that some small fraction $\epsilon_{\mathrm{f}}$ of the radiated luminosity $L_{\mathrm{r}}$ can couple to the surrounding gas in the form of feedback energy, viz. $\dot{E}_{\text {feed }}=\epsilon_{\mathrm{f}} L_{\mathrm{r}}$ In accordance with our previous studies of galaxy merger simulations we take $\epsilon_{\mathrm{f}}=5 \%$. This value governs the normalization of the $M_{\mathrm{BH}}-\sigma$ relation, and $\epsilon_{\mathrm{f}}=5 \%$ brings it into agreement with current observations (Di Matteo et al. 2005). We note that $\epsilon_{\mathrm{f}}$ is effectively the only free parameter in our black hole model. After fixing it to reproduce the normalization of the observed $M_{\mathrm{BH}}-\sigma$ from the galaxy models we do not vary it in any of our cosmological simulations.

For simplicity, we deposit the feedback energy isotropically in the region around the black hole. Lack of spatial resolution precludes us from considering mechanical modes of releasing the energy, e.g. in the form of a jet. However, we note that is is plausible that such other forms of energy are thermalized eventually as well, and that the final impact of the feedback depends primarily on the total amount of energy released and less on the form it is released in. This is likely to be true generally, provided that the energy (or momentum) is imparted to the surrounding gas on length scales small and time scales short compared with those that characterize the host galaxy. In that event, the impact of black hole feedback will be explosive in nature and, indeed, the blowout phase of evolution in our simulations is well-described by a generalized Sedov-Taylor blast-wave solution (Hopkins et al. 2006b; Hopkins \& Hernquist 2006). In any case, we emphasize that despite an isotropic release of the energy, the response of the gas can still be decidedly anisotropic, e.g. when a dense gas disk is present that channels the gas response into a collimated outflow.

The idea that we follow with our feedback modeling here is the rapid accretion phases of BHs at times close to their critical growth phases. Such 'quasar' phases are typically relatively short-lived and require galaxy mergers to produce the strong gravitational tidal forcing necessary for sufficient nuclear gas inflow rates. It is presently unclear whether the accretion disks in such modes actually produce mechanical jets of release their feedback in another way. It is however evident that at low redshift (e.g.; $z<1$ which we are not studying directly here) that when $\mathrm{BHs}$ that are embedded in the hot gas atmospheres of large groups and clusters channel their energy in mechanical feedback in the form of relativistic jets, gen- 
Di Matteo et al.

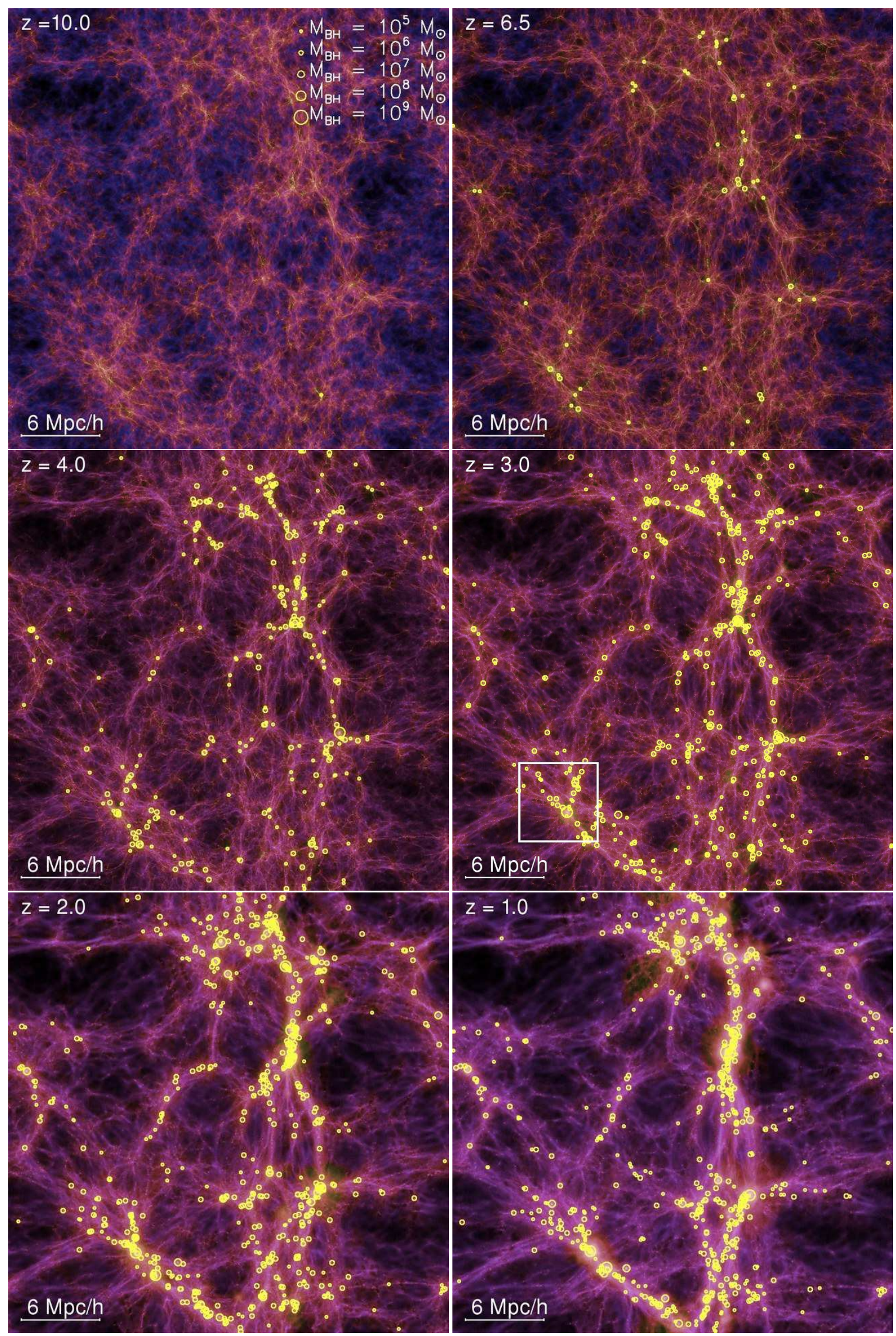

FIG. 1.- Projected baryonic density field in slices of thickness $5000 \mathrm{~h}^{-1} \mathrm{kpc}$ through our high resolution simulation, color-coded by temperature and with brightness proportional to the logarithm of the gas density. Each panel shows the same region of space at different redshifts, as labeled. The circles mark the positions of the black holes, with a size that encodes the BH mass, as indicated in the top left panel. 
TABLE 1

NUMERICAL PARAMETERS OF COSMOLOGICAL SIMULATIONS WITH BHS

\begin{tabular}{ccccccc}
\hline \hline Run & $\begin{array}{c}\text { Boxsize } \\
h^{-1} \mathrm{Mpc}\end{array}$ & $N_{p}$ & $\begin{array}{c}m_{\mathrm{DM}} \\
h^{-1} \mathrm{M}_{\odot}\end{array}$ & $\begin{array}{c}m_{\text {gas }} \\
h^{-1} \mathrm{M}_{\odot}\end{array}$ & $\begin{array}{c}\epsilon \\
h^{-1} \mathrm{kpc}\end{array}$ & $z_{\text {end }}$ \\
\hline D4 & 33.75 & $2 \times 216^{3}$ & $2.75 \times 10^{8}$ & $4.24 \times 10^{7}$ & 6.25 & 0.00 \\
D6 (BHCosmo $)$ & 33.75 & $2 \times 486^{3}$ & $2.75 \times 10^{7}$ & $4.24 \times 10^{6}$ & 2.73 & 1.00 \\
E6 & 50 & $2 \times 486^{3}$ & $7.85 \times 10^{7}$ & $1.21 \times 10^{7}$ & 4.12 & 4.00 \\
\hline
\end{tabular}

erating buoyantly rising radio bubble. In Sijacki et al. (2007) we consider this form of feedback mode.

An important remaining question in our model concerns the ultimate origin of the BHs. Since our accretion rate estimate can only grow a BH that already exists, we assume that a physical process that produces small seed BHs is operating sufficiently efficiently that effectively all halos above a certain threshold mass contain at least one such seed BH. Whether or not they can then grow to larger masses by gas accretion will be determined by the local gas conditions, as described in our model above. In order to achieve such a seeding at a technical level in cosmological runs we use an on-the-fly 'friends-of-friends' group finder algorithm which is called at intervals equally spaced in the logarithm of the scale-factor $a$, with $\Delta \log a=\log 1.25$. This provides the locations and mass of all halos in the simulation. If a halo is more massive than our threshold and does not contain any black hole yet, we endow it with one by converting its densest gas particle into a sink particle with a seed black hole mass of $10^{5} h^{-1} \mathrm{M}_{\odot}$. The further growth of the black hole sink can then proceed by gas accretion, at a rate that depends sensitively on the local conditions, or by mergers with other black hole sink particles. The total cumulative black hole mass introduced in this way as seeds is negligible compared to the mass growth by gas accretion. We note that being able to run a fast, parallel 'friends-of-friends' algorithm on the fly during simulations is an important technical prerequisite of our technique.

Further motivation for this choice of seeding procedure is based on the currently proposed scenarios for the seed black holes in galaxies. To grow a supermassive black hole to a mass of $\sim 10^{9} \mathrm{M}_{\odot}$ in less than a billion years, as required by presence of the $z=6 \mathrm{SDSS}$ quasars, may require (1) the catastrophic collapse of a supermassive star that forms a large initial black hole of mass $10^{4}-10^{6} \mathrm{M}_{\odot}$ (Carr \& Rees 1984; Bromm \& Loeb 2003; Begelman et al. 2006), or (2) alternatively, smaller black hole seeds $\left(M \sim 10^{2} \mathrm{M}_{\odot}\right)$ may form from the first PopIII stars at $z \sim 30$ and grow exponentially from then on (Abel et al. 2002; Bromm \& Larson 2004; Yoshida et al. 2006). In our simulations, black hole seeds of mass $M=10^{5} h^{-1} \mathrm{M}_{\odot}$ are introduced into galaxies as they initially reach $M_{\text {halo }}=10^{10} h^{-1} \mathrm{M}_{\odot}$. This choice is a good approximation to what is expected for both of the hypotheses outlined above. For (1) this is roughly in the correct range; whereas for (2) Eddington growth predicts that the black hole has grown to roughly these values by the time of collapse of $M \sim 10^{10} h^{-1} \mathrm{M}_{\odot}$ perturbations, which occurs at $z \sim 10$ in our standard cold dark matter scenario. Additionally, although not required, this value of the initial black hole mass to galaxy ratio fits the observed relations at low redshift (Magorrian et al. 1998; Ferrarese \& Merritt 2000). It is important to note that the dominant growth of black holes always occurs in exponential Eddington phases induced by gas accretion so that our results are rather insensitive to the specific choice of the seed mass.
When galaxies and their surrounding dark halos merge to form a single dark matter halo, their central black holes are also expected to merge eventually, so hierarchical black hole mergers contribute to the growth of the central black holes. Whether the forming black hole binaries can really coalesce efficiently is however a matter of debate. In a stellar environment, it has been argued that the binary hardens only very slowly (Begelman et al. 1980; Milosavljević \& Merritt 2003), while in gaseous environments binaries may coalesce rapidly owing to strong dynamical friction with the gas (Makino \& Funato 2004; Escala et al. 2004). In our galaxysized simulations, and even more so in the cosmological boxes, it is not possible to treat in detail the problem of binary hardening, nor to directly calculate the ejection of black holes by gravitational recoil, or by three-body sling-shot ejection of black holes in triple systems. Because galaxies have typically large central concentrations of gas we instead assume that two black hole particles merge quickly if they come within the spatial resolution of the simulation and their relative speed lies below the gas sound speed. In practice, this means that two sink particles that fulfill these conditions are merged into a single $\mathrm{BH}$ particle, with their masses combined.

\subsection{Simulation runs}

Cosmological simulations which include quasar formation must model sufficiently large volumes to sample a representative part of the universe, but also have high enough resolution to model the full hydrodynamics. This is a substantial challenge, given that the brightest quasars at $z \sim 6$ have a low space density and are believed to reside in fairly massive dark matter halos of mass $M \sim 10^{11}-10^{12} \mathrm{M}_{\odot}$, or even larger. At redshifts $z=2-3$, quasars have a much larger space density, comparable to $L^{*}$ galaxies at $z=0$.

The strategy we choose here is model the universe with a periodic box of moderate size $33.75 h^{-1} \mathrm{Mpc}$ that is homogeneously sampled with particles, and which we simulate with $2 \times 486^{3}$ particles, one of the highest resolutions so far achieved in a full cosmological hydrodynamical calculation of galaxy formation. In this paper, we refer to this largest simulation among our simulation set as the BHCosmo run. We will also compare it with two additional simulations which differ in mass and spatial resolution, and/or box size, to test for resolution effects.

The fundamental numerical parameters of our simulation runs are listed in Table 11 where $N_{p}$ is the number of dark matter and gas particles, $m_{\mathrm{DM}}$ and $m_{\mathrm{gas}}$ are their masses (initial mass in the case of the gas). Finally, $\epsilon$ gives the comoving gravitational softening length, and $z_{\text {end }}$ the final redshift of the simulation.

For the physical problem at hand we prefer relatively high resolution in order to capture the physics in high density regions appropriately, but we also need a large volume to study the growth of deep gravitational potentials. Our choice of $33.75 h^{-1} \mathrm{Mpc}$ represents a compromise in this respect. While this box-size is too small to be evolved to redshifts lower than $z \simeq 1$ (otherwise the fundamental mode would become non-linear), it is sufficiently large to provide a representative model for $L_{\star}$-objects at higher redshift, even though very rare systems in the exponential tail of the mass function will not be sampled well. We also note that the choice of box-size and particle number in the BHCosmo run is such that the physical resolution at $z \sim 6$ is comparable to that in some of our previous works on galaxy mergers, namely runs which used only $N \simeq 10000$ particles for each galaxy. In this prior work, 


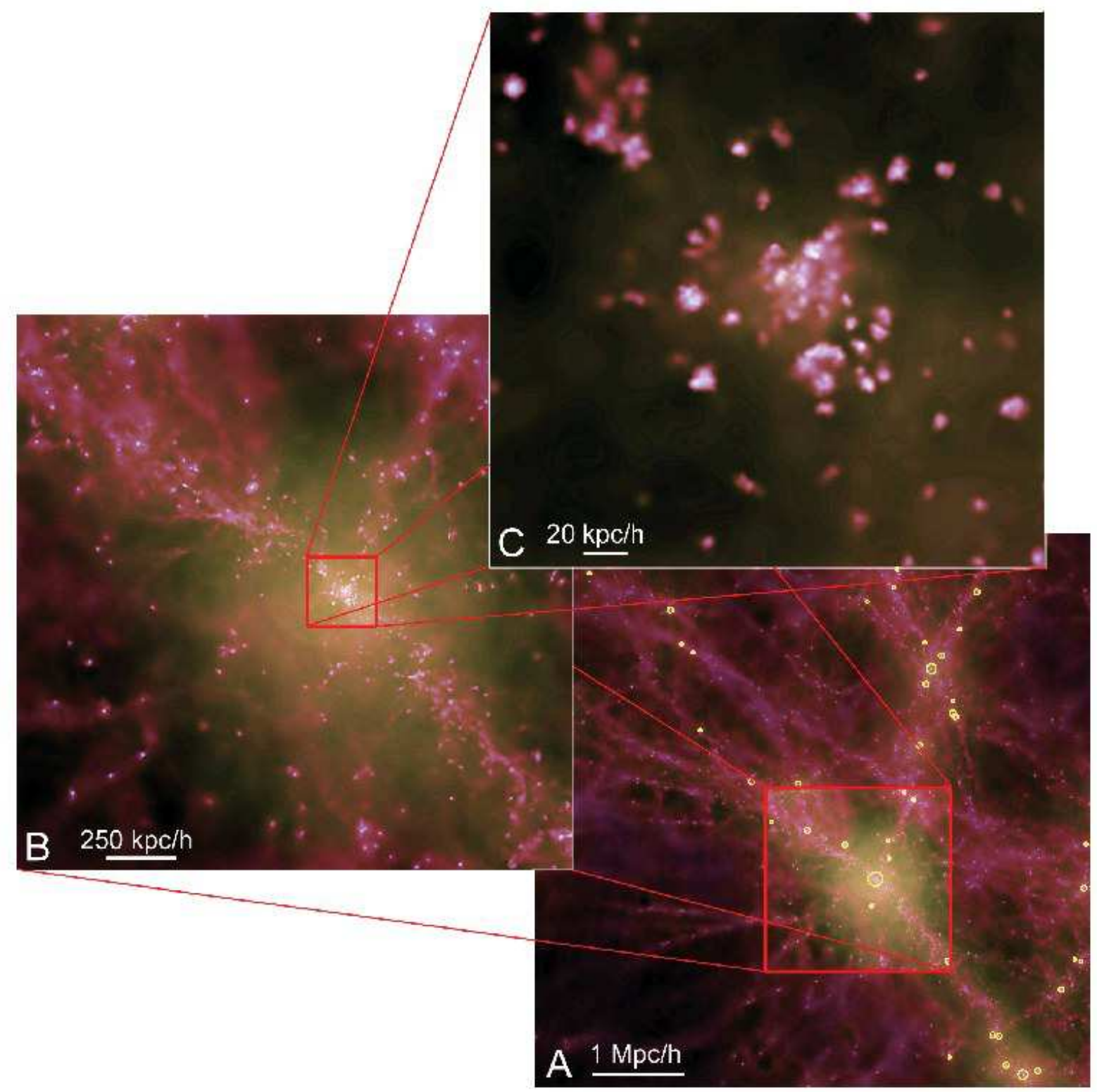

FIG. 2.- Three level zooms into the simulation region marked by the white rectangle in the $z=3$ panel of Fig 1 The three panels show the gas surface density, color-coded by temperature. The panels show slices of thickness $5000 \mathrm{~h}^{-1} \mathrm{kpc}$ and of decreasing width (from A to C) as we zoom into the region around a black hole of mass, $M_{B H} \sim 7 \times 10^{7}$. The yellow circles in the bottom right panel show the black holes in the region, with a symbol size that is related to the BH mass as in Fig. 1.

we have shown that despite the low resolution the results for the black hole mass growth agreed well with those obtained in runs with 128 times higher resolution, and can therefore be considered converged with respect to this quantity. This overlap in resolution between our cosmological runs and our previous work on isolated galaxy mergers gives us confidence that the results of our cosmological runs are not dominated by resolution effects, although it is clear that this needs to be tested separately. We remark that as part of our previous work on mergers we have run a suite of several hundred galaxy merger simulations (Robertson et al. 2006b), varying all the parameters describing star formation and feedback from supernovae and black hole growth and accretion, besides carrying out numerical resolution studies. The galaxy merger sim- ulations are clearly much better suited for investigating the full parameter space of our model, while for the cosmological runs we have to restrict ourselves to our default model owing to their much larger computational cost.

In addition to the above considerations, the choice of boxsize in our new simulations is also motivated by the set of simulations presented in Springel \& Hernquist (2003b). In fact, the BHCosmo run would be called 'D6' in their naming scheme. Being able to directly refer to their runs simplifies the comparison of the physical properties of simulations with and without black holes, e.g. with respect to the star formation history. 


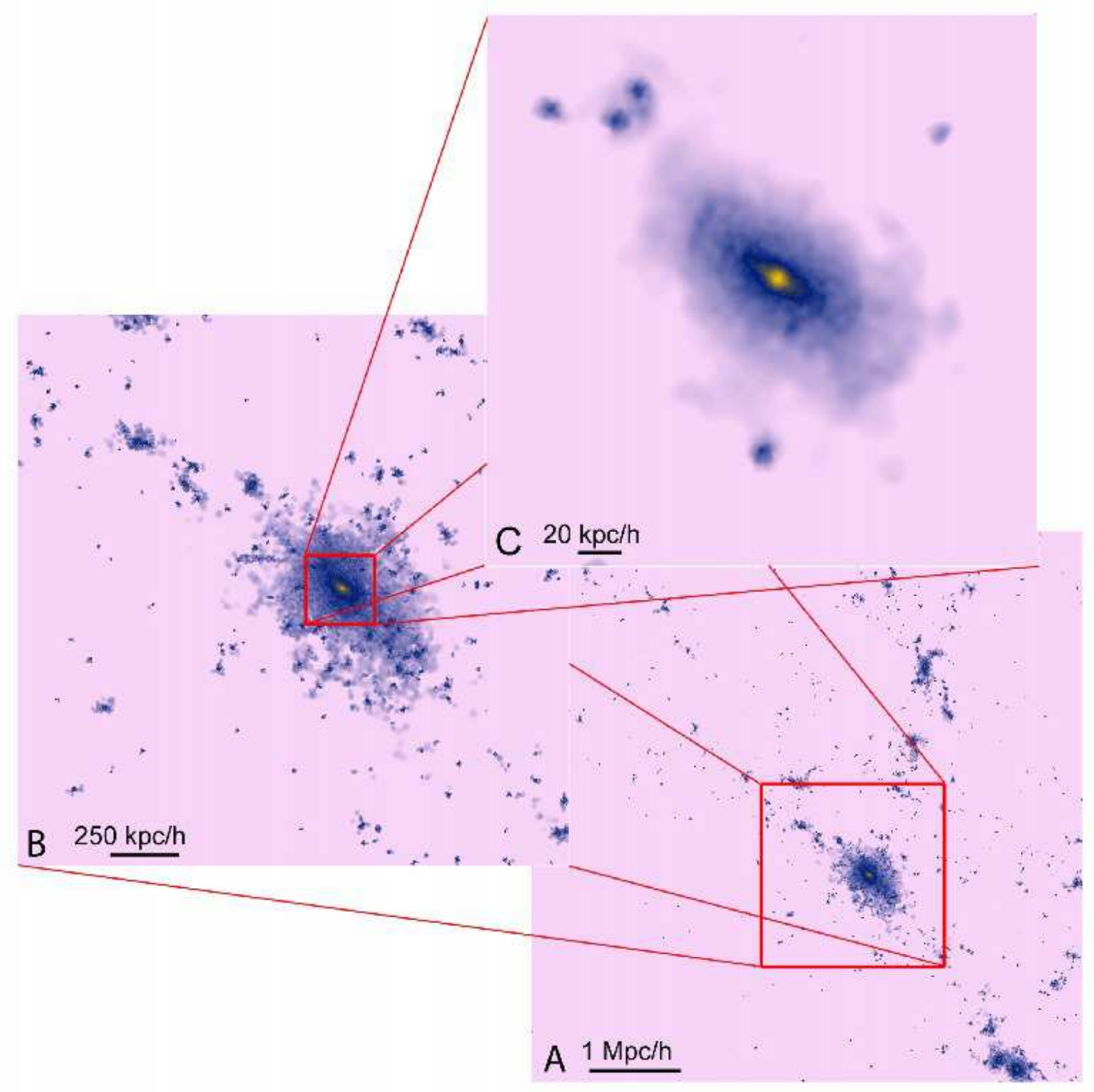

FIG. 3.- Stellar density in a three level zoom into the same regions shown in Figure 2 and which is marked by a white rectangle in the $z=3$ panel of Fig 1 In the most zoomed-in panel C, a stellar disk and a small bulge component can be seen for the central object.

\subsection{Visualization of the structure and black hole growth}

In Figure 1, we show slices through the BHCosmo simulation at a range of redshifts in order to visualize the evolution of the baryonic density field and the growth of black holes. The slice has a thickness of $5 h^{-1} \mathrm{Mpc}$ and shows the full box of size $33.75 h^{-1} \mathrm{Mpc}$ on a side. In each panel, the projected gas density field is color-coded according to the gas temperature, with the brightness of each pixel being proportional to the logarithm of the gas surface density. Circles of different size are drawn to mark the locations of $\mathrm{BHs}$ of different mass, as labelled.

The images show that black holes emerge in halos starting at high redshift (as early as $z \sim 12$ ) and subsequently grow by gas accretion, driven by gas inflows that accompany the hierarchical build-up of ever larger halos through merging. As the simulation evolves, the number of black holes rapidly in- creases and larger halos host increasingly more massive black holes. We note that the particular slice of the box shown in Figure 1 does not contain the largest black hole in the simulation's volume, which turns out to be located in the highest density region in the simulation. We will discuss this region separately in later sections.

By plotting the density field color-coded by temperature we can also see traces of heating effects from strong gas outflows, which are caused by black hole feedback and galactic winds from star formation. In our numerical mode, quasar-driven outflows occur once a central $\mathrm{BH}$ gas grown so much that its energy feedback in accretion phases is able to transfer sufficient energy to the remaining gas to unbind and expel part of it from the galaxy potential as a wind. This then terminates further strong growth of the $\mathrm{BH}$, which is key factor in establishing a self-regulated nature of the growth of black holes in 
our model, as we discussed in detail in our previous work on galaxy merger simulations.

To illustrate how well the mass resolution of the simulation captures details of galaxy formation sites, we zoom in onto a region of $6 h^{-1} \mathrm{Mpc}$ on a side at $z=3$ (as indicated by the box drawn in Fig 1), showing the gas density in Figure 2, and the stellar density of the same region in Figure 3 The middle panels (labeled B) in both figures show a second zoom-level into a region of $2 h^{-1} \mathrm{Mpc}$ on a side (indicated by the square in the A panels). Finally, the top panels show a further enlargement by a factor of 8 .

In accordance with our seeding procedure, black holes are located in the highest density regions. The more massive ones are found within the largest halos, which have also undergone more prominent star formation. Such a correspondence is expected in our model since large-scale gas inflows into the centers of halos lead both to star formation (and starbursts) as well as to nuclear black hole growth. In the highest level zoom of Figures 2 and 3, the central galaxy, with an extent of $\sim 50 h^{-1} \mathrm{kpc}$ (comoving), has a very rough disk-like morphology with a central stellar bulge. Nevertheless, it is clear that our cosmological simulations in general still have too low resolution for properly resolving galaxy morphologies. We also note that producing disk galaxies with the right size and abundance in cosmological hydrodynamical simulations is an essentially unsolved problem, and the outlook for obtaining a solution to this long-standing challenge has only slightly improved by recent works on disk galaxy formation (Robertson et al. 2004; Governato et al. 2007; Okamoto et al. 2007).

\subsection{The evolution of the global black hole mass density}

The black hole mass density at the present epoch is estimated from direct measurements of black hole masses in local galaxies (to establish, e.g., the $M_{\mathrm{BH}}-M_{\star}$ relationship), combined with a suitable integration over the galaxy luminosity function (Fabian 1999; Yu \& Tremaine 2002; Marconi et al. 2004). The local value thus obtained matches that of the total relic $\mathrm{BH}$ mass density estimated from a time integration of the luminosity output of active galactic nuclei and quasars at X-ray and optical wavelengths (Soltan 1982; Marconi et al. 2004; Shankar et al. 2004), or in a less model-dependent manner from an empirical determination of the bolometric quasar luminosity function (Hopkins et al. 2007b).

Figure 4 shows our simulation prediction for the global density $\rho_{\mathrm{BH}}$ and its evolution with redshift (thick black line). We find that the normalization of the black hole mass density is in agreement with the observational estimate of $\rho_{\mathrm{BH}}(0)=$ $4.6_{-1.4}^{+1.9} \times 10^{5} h_{0.7}^{2} \mathrm{M}_{\odot} \mathrm{Mpc}^{-3}$ of Marconi et al. (2004) and its extrapolation to $z \sim 3$, derived by exploiting hard $\mathrm{X}$-ray and optically selected AGNs and quasars. The grey area in Figure 4 shows the region delimited by observational constraints examined in detail in the literature (e.g Salucci et al. 1999; Marconi et al. 2004; Shankar et al. 2004). The agreement we see here is very reassuring. It shows that our black hole accretion and feedback model in the BHCosmo run is adequate and provides a realistic account for the dominant mode of global black hole growth in our universe.

The black hole mass density evolves rapidly at high redshift, increasing by four orders of magnitude between $z \sim 10$ to $z \sim 3$. While below this redshift, there is some further growth, it only accounts for roughly a doubling of the BH density down to $z=1$. This is corroborated by the bottom panel of Figure 4 , where we plot the history of the global black hole

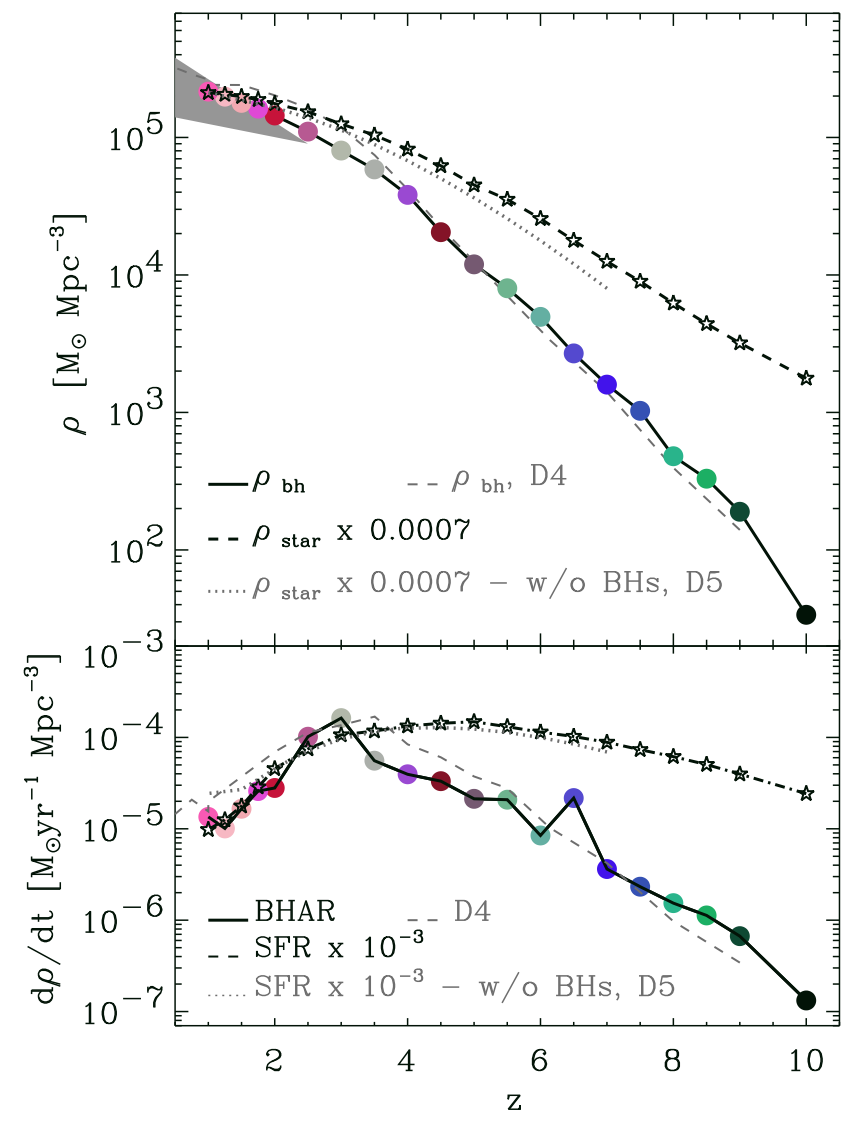

FIG. 4.- Top panel: The global black hole mass density evolution in the $B$ HCosmo/D5 run, shown by a solid line. The the star symbols and the dashed line give the corresponding stellar mass density evolution, multiplied by 0.0007 for easier comparison. The grey dotted line shows the stellar mass density in the D5 simulation which did not include black hole accretion and associated quasar feedback (Springel \& Hernquist 2003a). The thin dashed line shows the results from the lower resolution box, D4 described in Table 1 Different colors simply indicate the different redshifts consistent with the scheme used in other figures. The shaded grey triangle indicates observational constraints taken from the literature (Marconi et al. 2004; Shankar et al. 2004). Bottom Panel: The global history of the black hole accretion rate (solid line) and star formation rate (dot-dashed line with stellar symbols) densities. The SFR is rescaled by $10^{-3}$ for graphical clarity. In addition, we show the SFR history in the D5 simulations without black holes (grey dotted line). Most of the black hole and stellar mass is assembled by $z \sim 2-3$, but the peak in the BHAR density function is far more pronounced than that of the SFR density.

accretion rate (BHAR) density in our BHCosmo run. It is evident that the black hole mass growth occurs mainly by accretion above $z \sim 3$. Indeed, the BHAR density rises steadily at early times to a peak at $z \sim 3$, where it reaches a value $\sim 10^{-4} \mathrm{M}_{\odot} \mathrm{yr}^{-1} \mathrm{Mpc}^{-3}$. Below this redshift, it drops rather rapidly, becoming more than an order of magnitude lower by $z=1$. We note that the 'spike' seen in the BHAR density at $z \simeq 6.5$ is caused by a single object in the box, namely the rapid formation of the most massive black hole in the simulation at this epoch. We will discuss the history of the corresponding halo and its embedded black hole in more detail in $\S 5$.

\subsection{Black hole mass function and accretion rate function}

In Figure 5, we plot the black hole mass function at a number of different redshifts. We find that the final black hole 


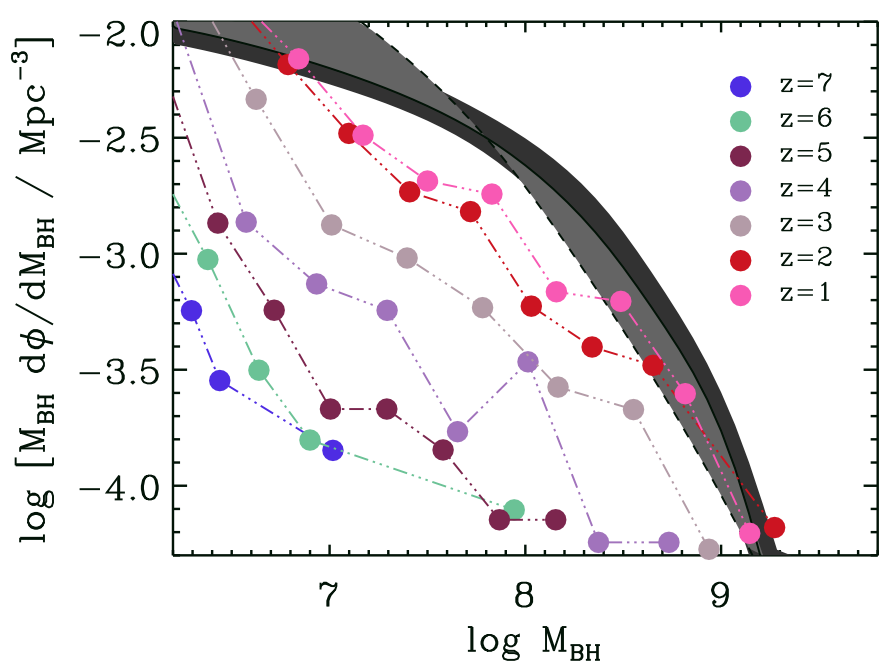

FIG. 5. - The evolution of the black hole mass function in the BHCosmo run. The different colors indicate different redshifts as labeled in the figure. For comparison, the dark grey shaded region shows the black hole mass function derived from local galaxies (Marconi et al. 2004). The light gray area adds the constraint from the integration of the hard X-ray luminosity function (Shankar et al.|2004; |Merloni|2004; | Marconi et al.|2004). The largest uncertainties are at the high and low mass end. The simulation results are in good agreement with the observed mass function at the high mass end, and in reasonable agreement at intermediate masses.

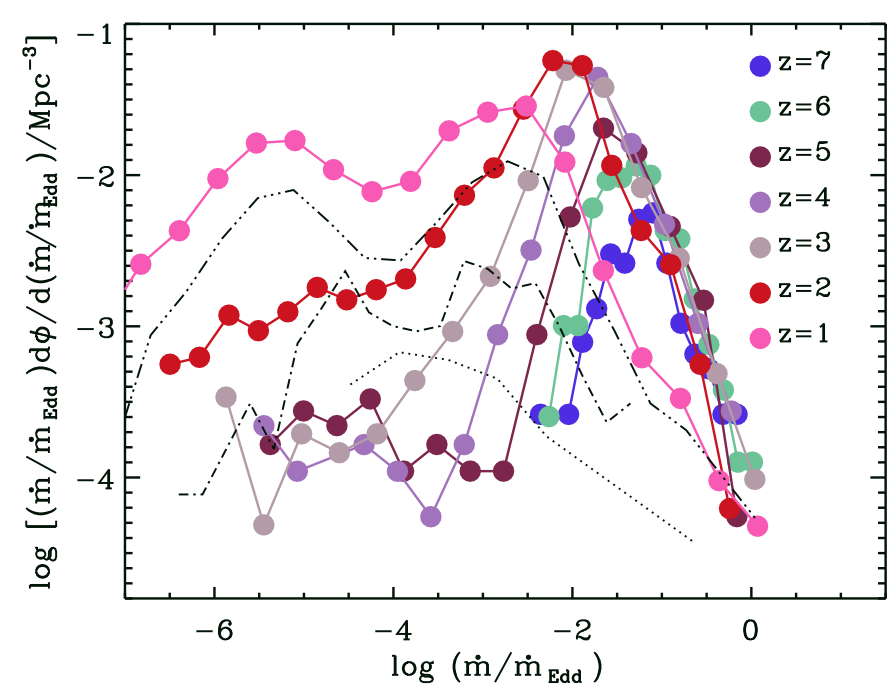

FIG. 6.- The time evolution of the accretion rate distribution as a function of the Eddington ratio, for the BHCosmo run. The different colors denote our measurements at different redshifts, as indicated in the legend. For the $z=1$ distribution function, we separately show three components corresponding to different regions of the black hole mass function. In particular, the dotted, dot-dashed, and dot-dot-dashed lines give the separate contributions from the three different mass bins $M_{\mathrm{BH}}>10^{8} \mathrm{M}_{\odot}, 10^{7}<M_{\mathrm{BH}} / \mathrm{M}_{\odot} \leq 10^{8}$ and $10^{6} \leq$ $M_{\mathrm{BH}} / \mathrm{M}_{\odot}<10^{7}$, respectively.

mass function in our simulation (for $z=1$ ) is is quite good agreement with the one measured locally, especially on the high-mass side. The $z=0$ constraint is indicated by the dark grey area taken from the compilation of Marconi et al. (2004), which is based on a combination of different observational data (Kochanek et al. 2001; Nakamura et al. 2003;
Bernardi et al. 2003; Sheth et al. 2003). There is a small deficit in our model at intermediate $\mathrm{BH}$ masses, but note that this will be filled in at least partly by the expected residual growth from $z=1$ to $z=0$. The light grey area adds an additional constraint for the contribution of relic AGN, derived from an integration of the the hard X-ray luminosity function (Shankar et al.2004). (Note that in this latter case the normalization of the mass function depends on the value assumed for the radiative efficiency when converting from the luminosity function to the mass function.)

As expected in a hierarchical formation scenario, the high mass end of the mass function shifts to larger masses with redshift. However, it is interesting that the mass function for high masses grows rather rapidly at early times relative to the low mass end. On the other hand, for redshifts $z \lesssim 2$, the high mass end is virtually fully assembled while the BH mass function continues to grow for low to intermediate masses, leading effectively to a steepening of the mass function for $z \lesssim 3$. This appears consistent with the emerging observational picture of the evolution of the supermassive black hole population according to which massive BHs $\left(M_{\mathrm{BH}} \gtrsim 10^{9} \mathrm{M}_{\odot}\right)$ are assembled early and are then likely undergoing comparatively passive evolution in the centers of large spheroids (Shields et al. 2003; Vestergaard 2004; Adelberger \& Steidel 2005). More generally, this phenomenon is described by the idea of an 'anti-hierarchical' black hole growth, or equivalently a 'downsizing' of black hole activity (Merloni 2004; Marconi et al. 2004), which is derived from constraints on the accretion history from X-ray luminosity functions.

Finally, in Figure 6, we show the evolution of the corresponding accretion rate distribution function for the black holes in our simulation, expressed in units of the Eddington rate. This function is strongly peaked at a few percent below the critical Eddington value, with most black holes accreting at $10^{-2} \lesssim \dot{m} / \dot{m}_{\text {Edd }} \lesssim 1$ at redshifts $z \gtrsim 6$. The distribution becomes wider and develops a small amplitude tail at low Eddington rates for $6<z<3$. Below $z \sim 3$ the peak of the accretion rate function shifts to $\dot{m} / \dot{m}_{\text {Edd }} \sim 10^{-2}-10^{-3}$ and an increasingly large population of sources is present accreting down to $10^{-6}$ Eddington. At $z \sim 1$ the distribution function is extremely wide and dominated by sub-Eddington (to severely sub-Eddington) sources, in terms of number. While hence the number of sources accreting at sub-Eddington rates increases sharply with decreasing redshift, the quasar-like population, i.e. the sources accreting close to Eddington, decreases with decreasing redshift, with a maximum at $z \sim 3$. For $z \sim 1$, we also include in Figure 6 measurements of the separate contributions to the accretion rate function from three different black hole mass ranges. The dotted line gives the Eddington ratio distribution for $M_{\mathrm{BH}}>10^{8} \mathrm{M}_{\odot}$, and the dash-dotted and dot-dot-dashed lines are for masses $10^{7}<M_{\mathrm{BH}} / \mathrm{M}_{\odot} \leq 10^{8}$ and $10^{6} \leq M_{\mathrm{BH}} / \mathrm{M}_{\odot}<10^{7}$, respectively.

Taken together, the evolution of the black hole mass and accretion rate functions implies that most massive black holes assemble early and do so at close to their critical rate. At low redshift, progressively lower luminosities and lower mass systems start dominating the black hole activity. This is in accord with recent results from studies of the hard X-ray luminosity function of quasars and AGN, e.g. typical Seyfert-like objects with $M_{\mathrm{BH}} \sim 10^{7}-10^{8} \mathrm{M}_{\odot}$ accreting at a few percent of the Eddington rate (e.g. Ueda et al. 2003; Hasinger et al. 2005). 

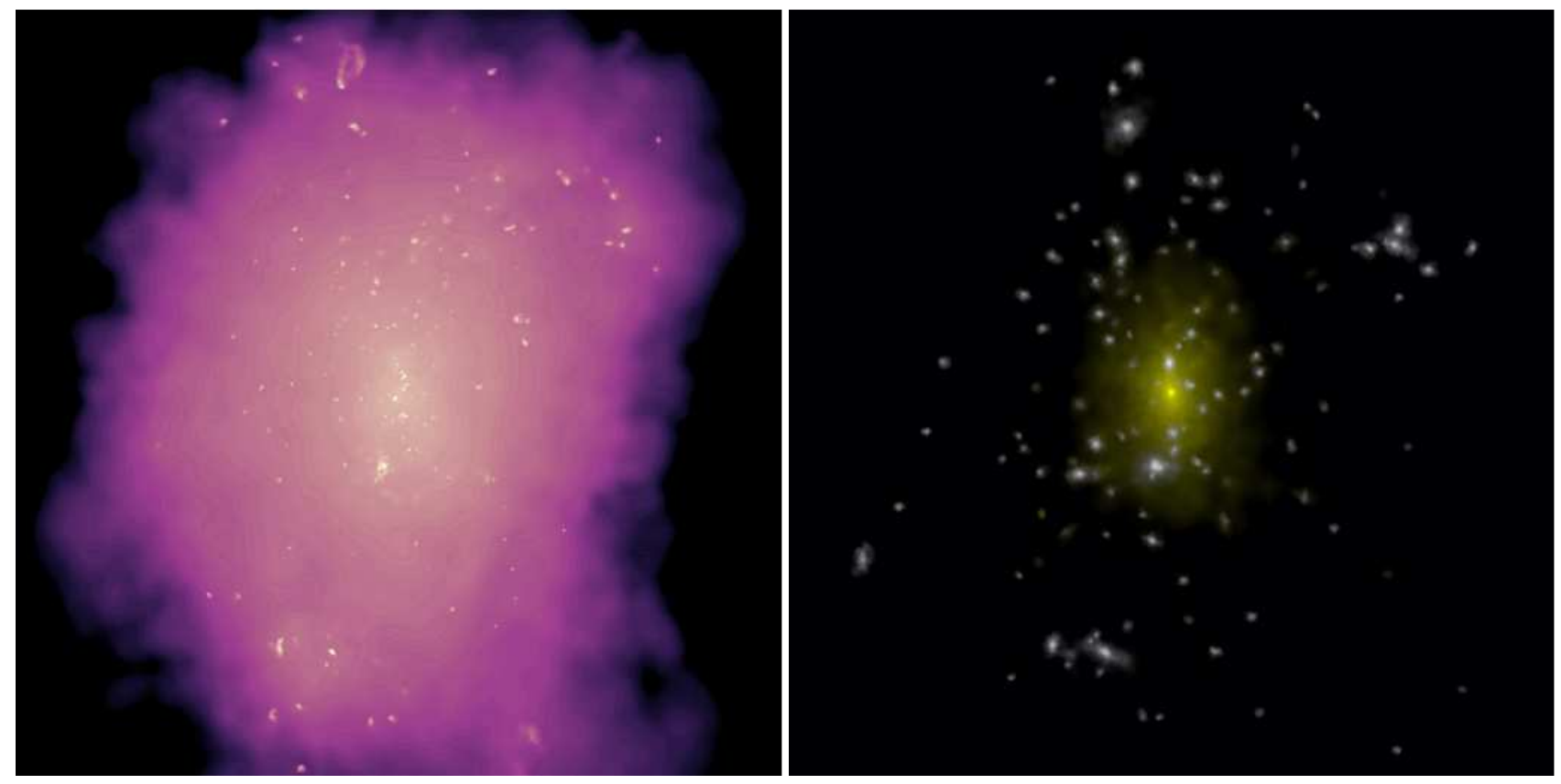

FIG. 7.- An illustration of a large group in the BHCosmo run at $z=1$. Left panel: the gas density distribution. Right panel: the stellar distribution. We have run a sub-group finder to identify all the systems (galaxies) within each halo and analyze their properties accordingly. The stellar system shown in yellow on the right hand side is the main galaxy within this large group, while the satellite galaxies are shown in grey. The images are $400 \mathrm{kpc}$ on a side.

\subsection{Comparison of the histories of black hole growth and star formation}

In Figure 4, we have already shown and discussed the evolution of the black hole mass and accretion rate densities. In the same Figure, we show corresponding results for the evolution of the stellar mass density, $\rho_{*}$, and the star formation rate (SFR) density, with their normalizations rescaled by factors of $7 \times 10^{-3}$ and $10^{-3}$, respectively, for plotting purposes. For comparison, we also include results for the D5 simulation of Springel \& Hernquist (2003b) which did not include black holes and any associated accretion or feedback processes (dotted grey lines).

We can see that $\rho_{*}$ far exceeds $\rho_{\mathrm{BH}}$ at all redshifts, with $\rho_{*}$ evolving less strongly with redshift than $\rho_{\mathrm{BH}}$ for $z \gtrsim 3$. At early times, $\rho_{\mathrm{BH}}$ rises more rapidly than the star formation density, while it tracks its evolution below this redshift. If we parameterize the ratio of $\rho_{\mathrm{BH}} / \rho_{*}$ by an evolutionary factor $(1+z)^{\alpha}$ we find that its evolution is approximately given by

$$
\rho_{\mathrm{BH}} / \rho_{*} \sim\left\{\begin{array}{ll}
\phi_{3}(1+z)^{-3} & \text { if } z \geq 3.5 \\
\phi_{1}[(1+z)]^{-0.6} & \text { if } z<3.5
\end{array},\right.
$$

with $\phi_{3}=5 \times 10^{-2}$ and $\phi_{1}=2.2 \times 10^{-3}$. Accordingly, up to $z \sim 3$, the evolution of the star formation rate density is considerably shallower than that of the black hole accretion rate density. Below this redshift, the BHAR and SFR densities closely track each other. As a result, the BHAR density has much more pronounced peak, which we find to lie at slightly lower redshift than the peak of the SFR. Parameterizing the evolution of the BHAR and SFR density ratio with a power law in $(1+z)$ leads to

$$
\dot{\rho}_{B H} / \dot{\rho}_{*} \sim\left\{\begin{array}{ll}
\dot{\rho}_{3}(1+z)^{-4} & \text { if } z \geq 3 \\
\dot{\rho}_{1}[(1+z)]^{-0.1} & \text { if } z<3
\end{array},\right.
$$

with $\dot{\phi}_{3}=0.2$ and $\dot{\phi}_{1}=10^{-3}$.

Note that Equations (1) and (2) are only meant to provide approximate scalings for our results from the simulations. The important point we want to emphasize is that our results imply a different and much stronger evolution of the black hole mass and accretion rate densities at high redshift relative to the stellar density and star formation rate density. The black hole mass density tends to be assembled later than the stellar mass, despite the growth of (a small number of) very massive BHs already at high redshift. However, for $z \sim 3$ and below, our models predict that the black hole mass and stellar mass densities, as well as the BHAR and SFR densities, closely track each other allowing only for small amounts of relative evolution between the two.

Another important point from Figure 4 is that the feedback we associate with black hole accretion does not significantly affect the global assembly of stellar mass. The peak of the SFR density is unaffected by the inclusion of BH feedback, but the drop in SFR density $(z<3)$ is slightly more abrupt in the simulations with black holes. This effect becomes more pronounced at $z \sim 1$, the final redshift for our simulation. At still lower redshift, we expect that BH feedback will become important in regulating the cooling and star formation in very massive halos. This is then ascribed not to quasar growth but rather to a 'radio mode'. We explore this different feedback channel in a companion paper by Sijacki et al. (2007).

\section{THE $M_{\mathrm{BH}}-\sigma$ AND $M_{\mathrm{BH}}-M_{*}$ RELATIONS \\ 4.1. Identification of groups and subgroups}

As a prerequisite for being able to study correlations between black hole and host galaxy properties in our simulation we first need to apply a suitable group finding algorithm that reliably identifies the stellar mass associated with the different galaxies. Note that especially the more massive halos identified by our basic friends-of-friends grouping algorithm used 


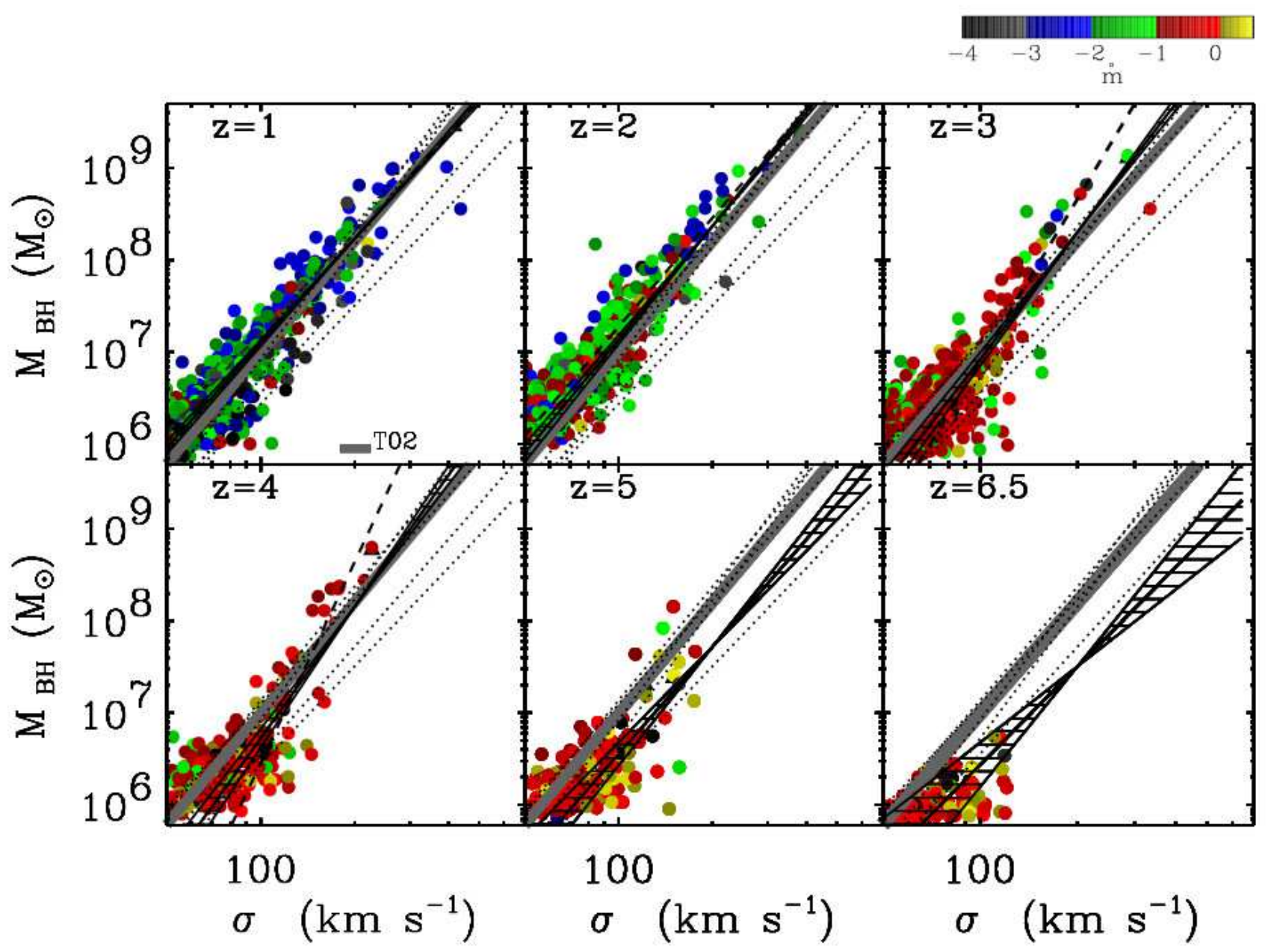

FIG. 8.- The evolution of the $M_{\mathrm{BH}}-\sigma$ relation in the BHCosmo simulation. The masses of BHs and the projected stellar velocity dispersions within the half mass radius $\left(R_{e}\right)$ have been measured in our simulated galaxies and are plotted at $z=1,2,3,4,5$, and 6.5 . We compared our measurements at all redshifts with the best-fit to the local $M_{\mathrm{BH}}-\sigma$ relation of Tremaine et al. (2002, hereafter T02), which is shown as a thick gray line. Linear regression fits to our simulated BHs are shown by solid lines at each redshift, with $1-\sigma$ errors indicated by the hatched regions. For ease of comparison, the dotted lines in each panel show the best fit relations at all redshifts. The points are color-coded according to their accretion rates in units of Eddington, as indicated in the color bar at the top right hand corner of the figure.

for finding virialized objects often contain a number of galaxies. This is illustrated in Figure 7, where we show a large cluster-sized group selected from the $z=1$ output of the $\mathrm{BH}$ Cosmo simulation. The panel on the left shows the gas density distribution in this large group, while the panel on the right hand side displays the stellar distribution. It is evident that the group contains several, gravitationally bound galaxies.

Our sub-group finder identifies all the galaxies within each group. Our method to identify galaxies within a given group is based on a variant of the SUBFIND algorithm (Springel et al. 2001). We first compute an adaptively smoothed baryonic density field for all stars and gas particles, allowing us to robustly identify centers of individual galaxies. We find the extent of these galaxies by processing the gas and star particles in the order of declining density, adding particles one by one to the galaxies attached to the galaxy to which its near- est denser neighbor already belongs (see also Nagamine et al. 2004). Note we are interested in the stellar and gas content of galaxies and associating the gaseous component to galaxies, as they typically they contain very dense star-forming gas, makes the method very robust in finding galaxies. This allows us to compute physical properties such as stellar mass, star formation rate, stellar velocity dispersion, metallicity, black hole mass and $\mathrm{BH}$ accretion rate separately for each galaxy. As an illustration, Figure 7 shows the stellar distribution associated with the largest galaxy in the group (which also hosts the most massive BH in the group) in yellow.

For each galaxy/subgroup that contains stars and a black hole, we calculate the projected (spherically averaged) halfmass effective radius, $R_{e}$, and the mass-weighted stellar velocity dispersion $\sigma$ within $R_{e}$. Note however that in order to make the measurements of $\sigma$ and $R_{e}$ somewhat accurate we 


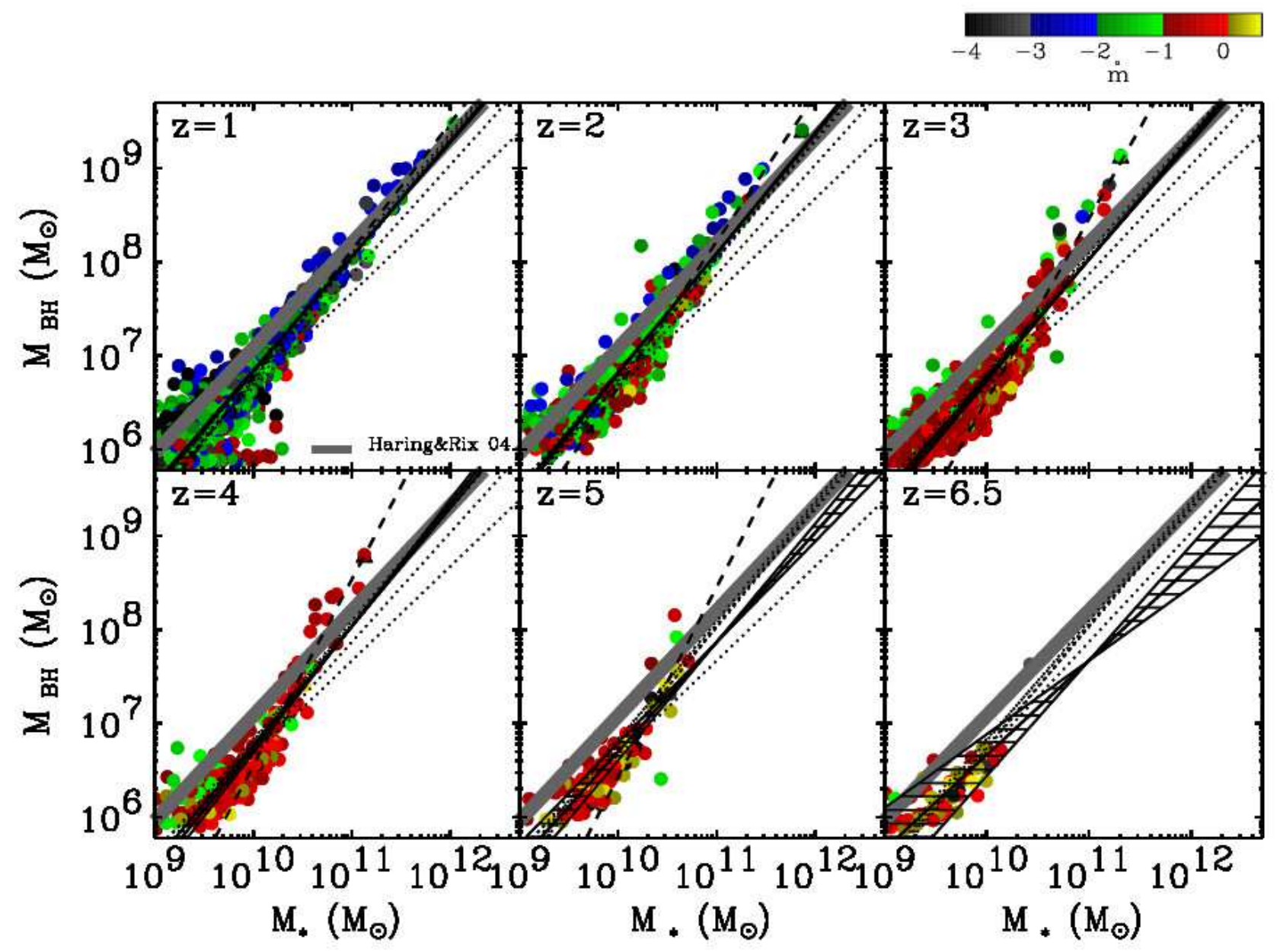

FIG. 9.- The evolution of the $M_{\mathrm{BH}}-M_{*}$ relation in the BHCosmo simulation. The masses of BHs and the corresponding stellar mass have been measured in our simulated galaxies and are plotted at $z=1,2,3,4,5$, and 6.5 . They are compared with the best-fit power law to the local $M_{\mathrm{BH}}-M_{*}$ relation by Häring \& Rix (2004), shown by the thick gray line. Our fits to the simulation results at each redshift are shown as solid black lines. The relation is tight with small scatter $(1-\sigma$ uncertainty ranges are plotted as hatched regions but are hardly visible at low redshift). As in Figure 8 the points are color coded by accretion rate. The dotted lines show in each panel the best fit relation at the other redshifts.

only consider those objects that contain more than 100 stars particles within $R_{e}$. This determination of $\sigma$ closely resembles the procedure for measuring the velocity dispersion from observational data (Gebhardt et al. 2000), allowing for a direct comparison.

\subsection{The predicted $M_{\mathrm{BH}}-\sigma$ and $M_{\mathrm{BH}}-M_{*}$ relationships and their evolution}

Figures 8 and 9 plot the $M_{\mathrm{BH}}-\sigma$ and $M_{\mathrm{BH}}-M_{*}$ relations, respectively, for our simulated galaxies at redshifts $z=1,2$, 3, 4, 5 and 6.5 (from top left to bottom right). Each measurement is color-coded according to the accretion rate of the corresponding black hole. We find a strong power-law correlation between both the velocity dispersion and the stellar mass with the black hole mass. Furthermore, at $z=1$, these correlations agree very well with the ones observed at the present epoch (Ferrarese \& Merritt 2000; Gebhardt et al.
2000; Tremaine et al. 2002; Häring \& Rix 2004) over a large dynamic range. This is a remarkable confirmation of the basic merger-driven scenario for self-regulated $\mathrm{BH}$ growth that we previously explored in isolated high-resolution galaxy merger simulations. Based on the same model, and without any finetuning or change of the model parameters, we here obtain a good match to the observed $M_{\mathrm{BH}}-\sigma$ relation directly from simulations that start from cosmological initial conditions and self-consistently account for the hierarchical build up galaxies in a $\Lambda C D M$ universe.

\subsubsection{The evolution of $M_{\mathrm{BH}}-\sigma$}

At higher redshifts, and in particular for $z>3$, our results for the $M_{\mathrm{BH}}-\sigma$ relation shows some degree of evolution relative to the local relations. To compare with the local data as a function of time, we fit the $M_{\mathrm{BH}}-\sigma$ relation at each redshift 
TABLE 2

PARAMETERS OF BEST-FIT $M_{\mathrm{BH}}-\sigma$ RELATIONS

\begin{tabular}{|c|c|c|c|c|}
\hline $\mathrm{z}$ & slope (a) & normalization, (b) & scatter $\Delta$ & slope $\left(\mathrm{a}_{s}\right)$ \\
\hline $1 \ldots \ldots$ & $3.95 \pm 0.10$ & $8.29 \pm 0.03$ & 0.10 & 3.9 \\
\hline 2 & $4.01 \pm 0.15$ & $8.42 \pm 0.04$ & 0.16 & 4.1 \\
\hline 3 & $4.21 \pm 0.22$ & $8.32 \pm 0.07$ & 0.23 & 5.2 \\
\hline $4 \ldots \ldots$. & $4.54 \pm 0.35$ & $8.17 \pm 0.10$ & 0.36 & 6.7 \\
\hline $5 \ldots \ldots$ & $3.37 \pm 0.45$ & $7.70 \pm 0.13$ & 0.47 & - \\
\hline $6.5 \ldots .$. & $3.26 \pm 0.85$ & $7.50 \pm 0.25$ & 0.89 & - \\
\hline
\end{tabular}

with a simple power-law of the form

$$
\log \left(\frac{M_{\mathrm{BH}}}{\mathrm{M}_{\odot}}\right)=a \log \left(\frac{\sigma}{200 \mathrm{~km} \mathrm{~s}^{-1}}\right)+b .
$$

The best-fit relations thus obtained are shown in Figure 8 as solid black lines, with the dispersion indicated by hatched regions. The dotted lines are the best-fit relations for all redshifts combined. We compare with the observed relation as determined by T02, which is described by a slope $a=4.02$, a normalization $b=8.2$ and a dispersion $\Delta \sim 0.25-0.30$ (grey thick line in Fig. 8).

The constants $a$ and $b$ for our best-fit relations and their dispersions are tabulated in Table 2 for different redshifts. Note that we here do not attempt to assess the statistical significance of the correlations in detail as the sources of systematic errors in the numerical measurements of $\sigma$ and $M_{\mathrm{BH}}$ cannot be easily quantified in the simulations. In particular, the cosmological simulations cannot determine the morphological properties of galaxies and therefore do not provide a direct measure of spheroid masses or their velocity dispersions. Our fitting procedure is merely intended to provide a first characterization of the overall evolution of the slope and normalization of the relations in the simulation model. As Figure 8 and Table 2 indicate, the $M_{\mathrm{BH}}-\sigma$ relation predicted from our simulation is consistent with a slope $\sim 4$ at low redshift, as observed. At $z=3-4$, the slope appears to be slightly steeper and at $z=5-6$ slightly shallower, but the small number of systems at $z \sim 6$ makes the latter trend uncertain.

Inspection of Figure 8 shows a qualitative trend whereby the larger systems with high $\sigma \gtrsim 150 \mathrm{~km} \mathrm{~s}^{-1}$ (which are also those that are best resolved in our simulation) appear to populate the high mass end of the $M_{\mathrm{BH}}-\sigma$ relation already at $z>2-3$. The lower mass end of the $M_{\mathrm{BH}}-\sigma$ relation is then increasingly filled in towards $z \sim 1$. To illustrate this trend more explicitly, we perform a fit of the relation using only those systems with $\sigma \geq 150 \mathrm{~km} \mathrm{~s}^{-1}$; this is shown by the dashed line in Figure 8 . The slope $a_{s}$ obtained for these 'high' $\sigma$ black holes is typically steeper than for the full relation (our measured values for $a_{s}$ are listed in Table 2). At a fixed and relatively high $\sigma$, the mean $M_{\mathrm{BH}}$ is larger at $z \sim 3-4$ than at $z \sim 1$. This trend suggests that black hole growth predates the final growth of the spheroid potential at scales $\sigma \sim 170 \mathrm{~km} \mathrm{~s}^{-1}$, which is consistent with the recent measurements of the $M_{\mathrm{BH}}-\sigma$ relation in a sample of Seyferts at $z \sim 0.35$ by Woo et al. (2006). This relatively more efficient black hole growth at a fixed $\sigma$ at high redshift may thus be responsible for a mild change in the high mass end of the $M_{\mathrm{BH}}-\sigma$ relation. In the remainder of this section we will discuss possible additional dependences in the $M_{\mathrm{BH}}-\sigma$ (Hopkins et al. 2007a) relation which may produces a small change in the slope of the relation with time.

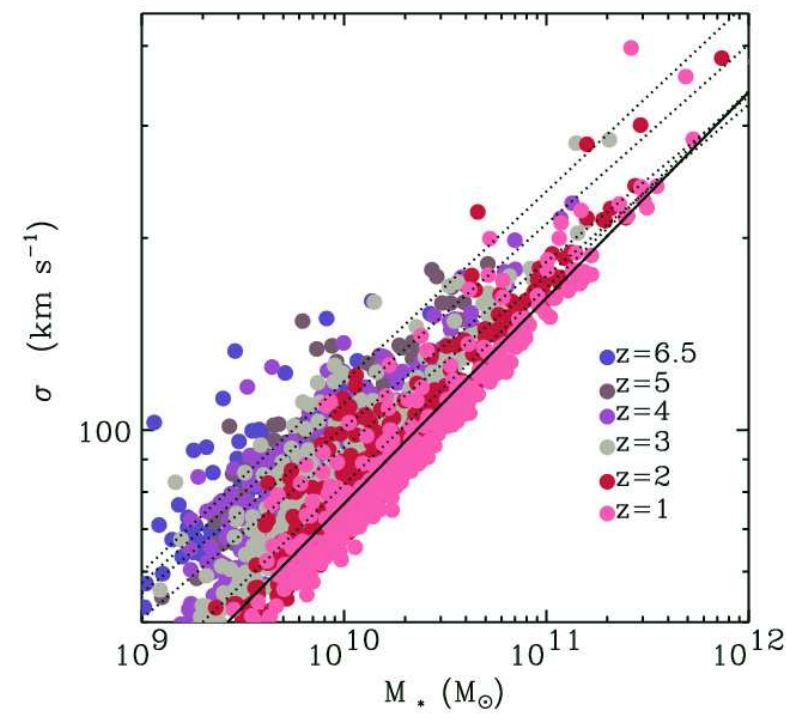

FIG. 10.- The stellar velocity dispersion $\sigma$ versus stellar $M_{*}$ at $z=1,2$, $3,4,5,6.5$ (indicated by different colors from blue to pink, the same ones as used in Figs. 4.6. The best-fit power-law to the trend is shown with a dotted line at each redshift, and with a solid line at $z=1$. The dispersion $\sigma$ at fixed $M_{*}$ increases with increasing redshift, which can be interpreted as a weak evolution in the Faber \& Jackson relation.

Note also that at $z \geq 4$ black holes are more likely to accrete close to their critical Eddington rates, as we showed earlier. In our models, the $M_{\mathrm{BH}}-\sigma$ relation is a natural consequence of the self-regulated growth of black holes (Di Matteo et al. 2005), where a black hole grows until its released energy is sufficient to expel the gas in its surroundings in a quasar driven wind, which then terminates nuclear accretion. For this reason, we expect the relation to show more scatter at times when most systems are still actively growing and accreting close to their Eddington values (see Table 2). This is expected as the primary path for assembly $\mathrm{BH}$ mass is via accretion during major mergers so that the relations converge and get increasingly tighter as galaxies undergo major mergers and continue to merge.

The overall normalization of the $M_{\mathrm{BH}}-\sigma$ relation evolves weakly with redshift when compared to the TO2 result for the present epoch. Below $z \sim 3$, there is virtually no evolution (bearing in mind the weak trend discussed above for $\sigma>150 \mathrm{~km} \mathrm{~s}^{-1}$ ), while we find a weak drift of the normalization beyond $z \sim 3$. A rough parameterization of this evolution in the normalization of the $M_{\mathrm{BH}}-\sigma$ relation is given by $M_{\mathrm{BH}} / \sigma^{4} \propto(1+z)^{\alpha}$ with $\alpha=-0.2$.

These results for an overall modest evolution in the normalization are in qualitative agreement with those from an analysis of isolated galaxy merger simulations by Robertson et al. (2006b). We note that in the latter study a redshift scaling of galaxy properties and a specific set of structural properties of the merging galaxies had to be assumed, which introduced an important systematic uncertainty. While having lower numerical resolution per merger, the simulation we analyze here eliminates this caveat by providing a fully self-consistent cosmological history for the formation and evolution of galaxies and their black holes (albeit at a lower spatial resolution). This provides an important confirmation of the analysis of Robertson et al. (2006b), who however did not find evi- 
TABLE 3

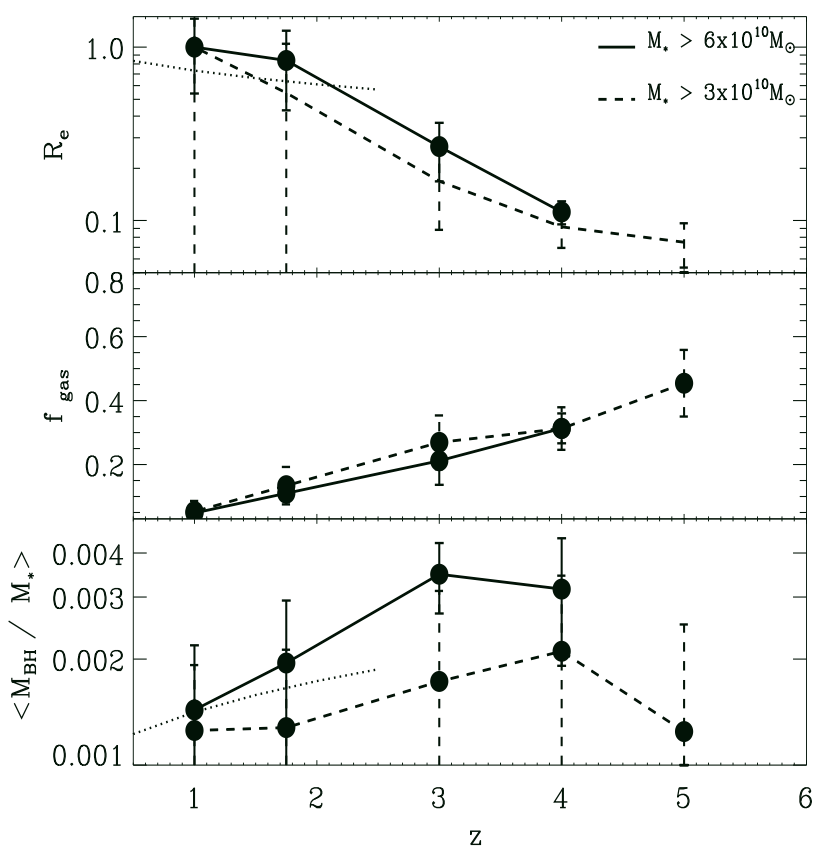

FIG. 11.- The redshift evolution of the projected half mass radius $R_{e}$ (top panel), cold gas fraction $f_{\text {gas }}$ (middle panel) and characteristic black hole to stellar mass ratio $\left\langle M_{\mathrm{BH}} / M_{*}\right\rangle$ (bottom panel), for systems with $M_{*}>3 \times$ $10^{10} \mathrm{M}_{\odot}$ (dashed lines) or $M_{*}>6 \times 10^{10} \mathrm{M}_{\odot}$ (solid) in the BHCosmo run. These threshold values were chosen to compare with the observed evolution determined by Truillo et al. (2006). The increase in cold gas content in high redshift progenitor hosts, and the trend to more compact systems with an increasing ratio of $M_{\mathrm{BH}} / M_{*}$ at a fixed stellar mass is consistent with the recent results of Truillo et al. (2006) and Peng et al. (2006), as well as the BHFP (Hopkins et al. 2007a).

dence for an evolution of the slope at the high mass end of the $M_{\mathrm{BH}}-\sigma$ relation.

\subsubsection{The evolution of $M_{\mathrm{BH}}-M_{*}$}

In Figure 9, we show the $M_{\mathrm{BH}}-M_{*}$ relation from the simulations at $z=1,2,3,4,5$ and 6.5 , alongside the local observational relation determined by Häring \& Rix (2004, thick grey lines). Our best-fit relation at each redshift is shown by a solid line while the dotted lines in each panel show results for the other redshifts. Table 3 gives the slope $c$, normalization $d$, and dispersion $\Delta_{m}$ for our best-fit relations of the form

$$
\log \left(\frac{M_{\mathrm{BH}}}{\mathrm{M}_{\odot}}\right)=c \log \left(\frac{M_{*}}{10^{11} \mathrm{M}_{\odot}}\right)+d .
$$

As before, our fitted values for $c$ and $d$ are intended to indicate general trends in the evolution rather than to be used as statistically rigorous measurements. The observed relationship (Häring \& Rix 2004) has a slope $c=1.12$ and normalization $d=8.2$.

Overall there appears to be only limited evolution in the $M_{\mathrm{BH}}-M_{*}$ relation, but there is a slight steepening at $z=2-4$. To highlight this trend, we restrict our fits to the high mass end with $M_{*} \geq 5 \times 10^{10} \mathrm{M}_{\odot}$ (dashed line in Fig. 9). In this range, the relation is significantly steeper, implying slopes $c_{s} \sim 1.9$ at $z=3-4$ and $\sim 1.5$ at $z \sim 2$. This is more significant than the evolution found in the slope of $M_{\mathrm{BH}}-\sigma$, and implies that
PARAMETERS OF BEST-FIT $M_{\mathrm{BH}}-M_{*}$ RELATIONS

\begin{tabular}{|c|c|c|c|c|}
\hline$z$ & slope $c$ & normalization $d$ & scatter $\Delta$ & $\mathrm{c}_{s}$ \\
\hline $1 \ldots \ldots$ & $1.18 \pm 0.02$ & $8.10 \pm 0.03$ & 0.03 & 1.2 \\
\hline & $1.23 \pm 0.03$ & $8.09 \pm 0.03$ & 0.04 & 1.5 \\
\hline $3 \ldots \ldots$. & $1.25 \pm 0.04$ & $8.04 \pm 0.04$ & 0.06 & 1.9 \\
\hline $4 \ldots \ldots$ & $1.30 \pm 0.05$ & $8.04 \pm 0.05$ & 0.07 & 1.9 \\
\hline 5. & $1.17 \pm 0.10$ & $7.90 \pm 0.10$ & 0.14 & 2.0 \\
\hline $6.5 \ldots$. & $1.01 \pm 0.22$ & $7.78 \pm 0.25$ & 0.34 & - \\
\hline
\end{tabular}

there is some evolution in the ratio of black hole mass to stellar mass relative to the local observations. More precisely, systems with $M_{*} \gtrsim 10^{10} \mathrm{M}_{\odot}$ have larger black hole masses at fixed $M_{*}$ than at $z=1$, where the ratio is in good agreement with the relation observed at the present epoch. This trend of an increasing $M_{\mathrm{BH}} / M_{*}$ ratio as a function of redshift appears consistent with the recent measurements of high redshift (up to $z \sim 3.5) \mathrm{BH}$ masses and host luminosities by Peng et al. (2006), as well as the BHFP (Hopkins et al. 2007a). We will further analyze this effect in $\$ 4.3$

\subsection{Evolution of $\sigma$, gas fraction, and $R_{e}$, at fixed stellar host mass}

We now analyze some of the physical properties of the host galaxies and their evolution with redshift to investigate the physical origin for the trends we have found in the $M_{\mathrm{BH}}-\sigma$ and $M_{\mathrm{BH}}-M_{*}$ relations. Figure 10 shows the stellar velocity dispersion $\sigma$ versus the stellar mass $M_{*}$ for each galaxy as a function of redshift. The dotted lines and the solid line (for $z=1$ ) show our best-fit relations. At a fixed $M_{*}$, the velocity dispersion $\sigma$ increases with increasing redshift. This is consistent with the results from the merger remnants in Robertson et al. (2006b) and reflects changes in the structural properties of stellar spheroids, which become smaller towards higher redshift.

In Figure 11, we show the projected half mass radius $R_{e}$, the cold gas fraction $f_{\text {gas }}$ (a proxy for the disk gas fraction), and the $\left\langle M_{\mathrm{BH}} / M_{*}\right\rangle$ ratio as a function of redshift. We show results for systems with $M_{*} \geq 3 \times 10^{10} \mathrm{M}_{\odot}$ (dashed lines) and $M_{*} \geq 6 \times 10^{10} \mathrm{M}_{\odot}$ (solid lines). We find higher cold gas fractions in higher redshift systems with $f_{\text {gas }} \propto(1+z)^{1.5}$. The increased gas content, dissipation rate in high redshift progenitor is consistent with them being more centrally concentrated, as shown by the trend of decreasing $R_{e}$ and increasing $\sigma$ with increasing redshift, leading to larger ratios $M_{\mathrm{BH}} / M_{*}$ at high redshifts, with $M_{\mathrm{BH}} / M_{*} \sim(1+z)^{0.5}$, at least over these ranges of $M_{*}$. Larger values of $\sigma$ at fixed $M_{*}$ at high redshift are also consistent with an inverse overall trend (e.g.; $(1+z)^{-0.2}$ ) in the evolution of the $M_{\mathrm{BH}}-\sigma$ relation.

The above results are consistent with the general picture for a merger-driven quasar growth we have developed based on our galaxy merger simulations (Di Matteo et al. 2005; Springel et al. 2005b; Robertson et al. 2006b; Hopkins et al. 2005, 2006a, 2007a), which also implies that the $M_{\mathrm{BH}}-\sigma$ and $M_{\mathrm{BH}}-M_{*}$ relations are connected to each other. This connection is most clearly demonstrated by the 'black hole fundamental plane' (BHFP) relation discussed by Hopkins et al. (2007a), which arises from the joint formation process of spheroids and massive black holes in mergers. Our cosmological simulations also should agree with the BHFP if most of the $\mathrm{BH}$ growth is associated with mergers. In Figure 12, we 

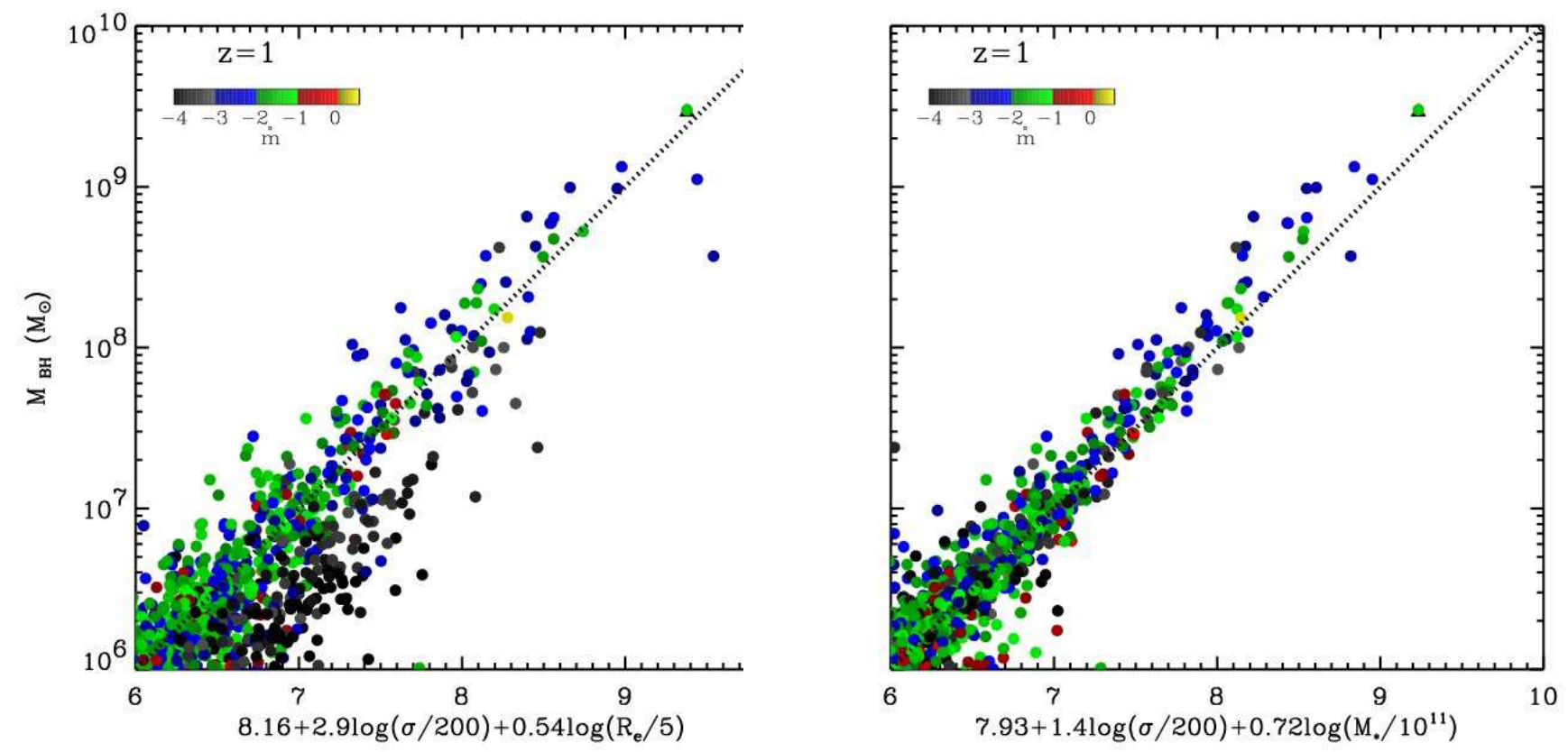

FIG. 12.- Simulation prediction for two projections of the 'Black Hole Fundamental Plane' (BHFP) relation at $z=1$, in terms of $\sigma$ and $M_{*}$ (left panel), and $\sigma$ and $R_{e}$ (right panel). We compare with the best-fit relations from Hopkins et al. (2007a), shown as dotted lines. The simulation agrees well with the conjecture of a BHFP, which confirms the overall trends we have found in the $M_{\mathrm{BH}}-\sigma$ and $M_{\mathrm{BH}}-M_{*}$ relations. This likely owes to the scatter in the measurements of both $\sigma$ and in particular $R_{e}$ which are noisy measurements in the simulations. The colors indicate accretion rate values (as in previous figures and indicated in the color bar).

show our simulation measurements for the BHFP and compare it to the the best-fit from Hopkins et al. (2007a), shown as a solid line. We find very good agreement with the predictions for the BHFP by Hopkins et al. (2007a), in both of its formulations, i.e. for $R_{e}$ and $\sigma$ or $M_{*}$ and $\sigma$, although the former shows a larger scatter than the latter, owing to the noisy measurements of $R_{e}$ in the cosmological simulation.

\section{THE FIRST AND THE MOST MASSIVE BLACK HOLES}

In the previous sections we have discussed the predictions from our simulation for the global history of black hole mass assembly in galaxies from the high redshift Universe to today. We have compared the predictions for the evolution of the black hole mass and accretion rate density to the history of the star formation rate density and discussed the growth of the black hole mass function. We have also discussed the $M_{\mathrm{BH}}-\sigma$ and $M_{\mathrm{BH}}-M_{*}$ relationships as a function of time, and examined which physical properties drive their cosmological evolution.

However, our simulation methodology not only allows us to make statistical statements about the black hole population. Rather, it can also be used to study the detailed growth history of individual black holes, from the moment they are seeded to today, which provides a particularly powerful way to follow the evolution of black holes over cosmic time. The gas which fuels black hole activity ultimately has its origins in the intergalactic medium, draining along filaments into forming galaxies. Because of this, $\mathrm{BH}$ radiative histories are directly linked to the formation of large-scale structure in the Universe, from supercluster scales down to the immediate environment of host galaxies. Being able to follow these large-scale processes and their impact on individual black holes self-consistently is the key to a qualitatively better level of understanding. For example, we have the tools to examine what turns a small black hole into a supermassive one at $z=6$, or whether some BHs grow hardly at all after this initial phase (as we shall see below in some examples). We can ask how quasar lifetimes are related to their clustering, how important $\mathrm{BH}$ mergers versus gas accretion are in the cosmological growth of BH mass, what $\mathrm{BH}$ light curves look like in detail over a Hubble time, and how specific outbursts correlate with galaxy and cluster merger and accretion events.

In future work we will return to address such questions in more detail; for example in Colberg et al. (2007) we present an extensive study of $\mathrm{BH}$ environments as the universe evolves. For now we will show that there are some striking inferences to be drawn from following the detailed histories of even a few black holes. As an example we choose to focus on the formation and fate of the first large black holes that form in our simulation, as well as the properties of their hosts and their descendants. This allows us to identify the prerequisite conditions for the growth of supermassive black holes already in the early universe.

Observations of luminous SDSS quasars at redshifts as high as $z \sim 6$ (Fan et al. 2003) present a number of challenges for models of high redshift quasar and galaxy formation. Their low space density suggests that they reside in the rarest dark matter density peaks at this early epoch, yet the apparent lack of companion galaxies in the field has been used to argue that these quasars reside in far more common halos (Carilli et al. 2004; Willott et al. 2005). Extensive follow-up observations of the highest redshift $(z=6.42)$ quasar, SDSS $\mathrm{J} 11148+5251$, are starting to constrain the properties of its host galaxy (Walter et al. 2004). CO observations indicate a relatively small stellar spheroid, however, the existence of $\mathrm{CO}$, iron and carbon emission indicates a heavily enriched 


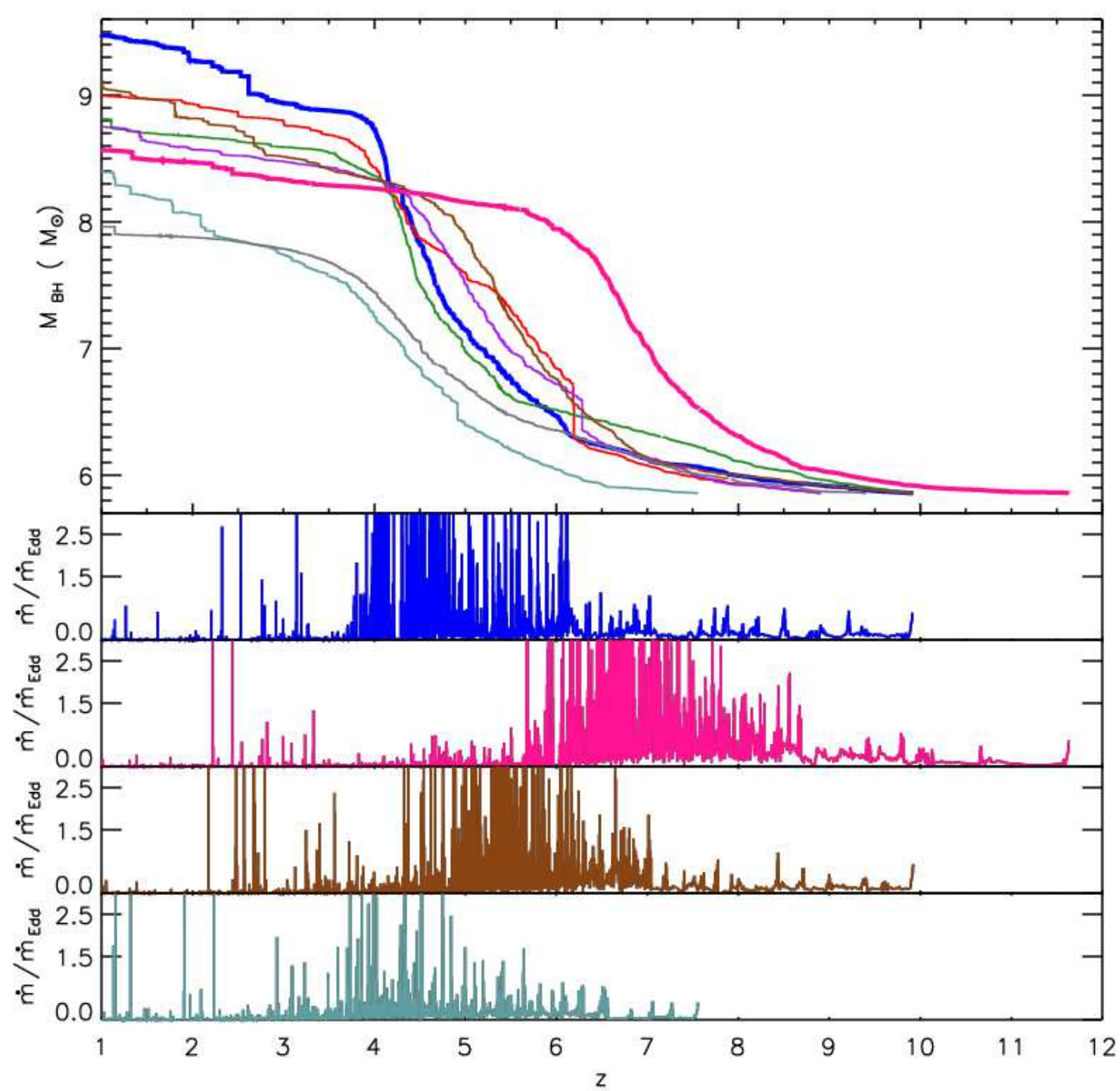

FIG. 13.- Individual mass assembly and accretion rate histories for the six most massive black holes and two intermediate mass BHs (chosen randomly) in the BHCosmo run. The top panel shows the black hole mass as a function of redshift while the bottom four panels give the detailed accretion rate history for the black holes with the corresponding colors in the top panel. The first supermassive black hole forming in the BHCosmo run, and the most massive one at the end of the run, are shown with thicker lines in pink and blue, respectively. From the bottom panels we see that phases of high Eddington accretion occur at different times of the history of different black holes.

ISM and vigorous star formation (Maiolino et al. 2005). In any case, rapid growth is clearly required to produce large supermassive black holes and spheroids in the $\sim 800$ million years available to $z \sim 6$. Unsurprisingly, the type of object associated with the 'first quasar' is therefore still a matter of debate.

The relatively small volume of our simulation prevents us from directly addressing the problem of the 'first quasars', simply because their space density is so low. Properly identifying one of the rare host systems requires at the very least box-sizes of $500 \mathrm{~h}^{-1} \mathrm{Mpc}$ (Springel et al. 2005d), or more adequately $1 h^{-1} \mathrm{Gpc}$ (Li et al. 2007). Nevertheless, our simulation allows us to examine the nature of the environments and the hosts where exponential growth of BHs can first occur at early times. This has some relevance to the problem of where the first quasars are likely to form.
In order to retrieve the required information for all the black holes in the simulation we have constructed full merger trees for each of them. This allows us to track the growth and accretion history of individual black holes of different masses, make detailed lightcurves from their accretion histories, and study the properties of their host galaxies.

\subsection{Individual BH mass and accretion rate as function of $z$}

In Figure 13, we show the accretion histories of a number of black holes in the BHCosmo run (top panel) and some examples of their corresponding accretion rate histories in the bottom four panels. The sample in the top panel consists of the six most massive black holes in the simulation and two randomly chosen ones with an intermediate mass at $z=1$. The four bottom panels show the accretion rate in units of Eddington for four black holes with corresponding line colors, 

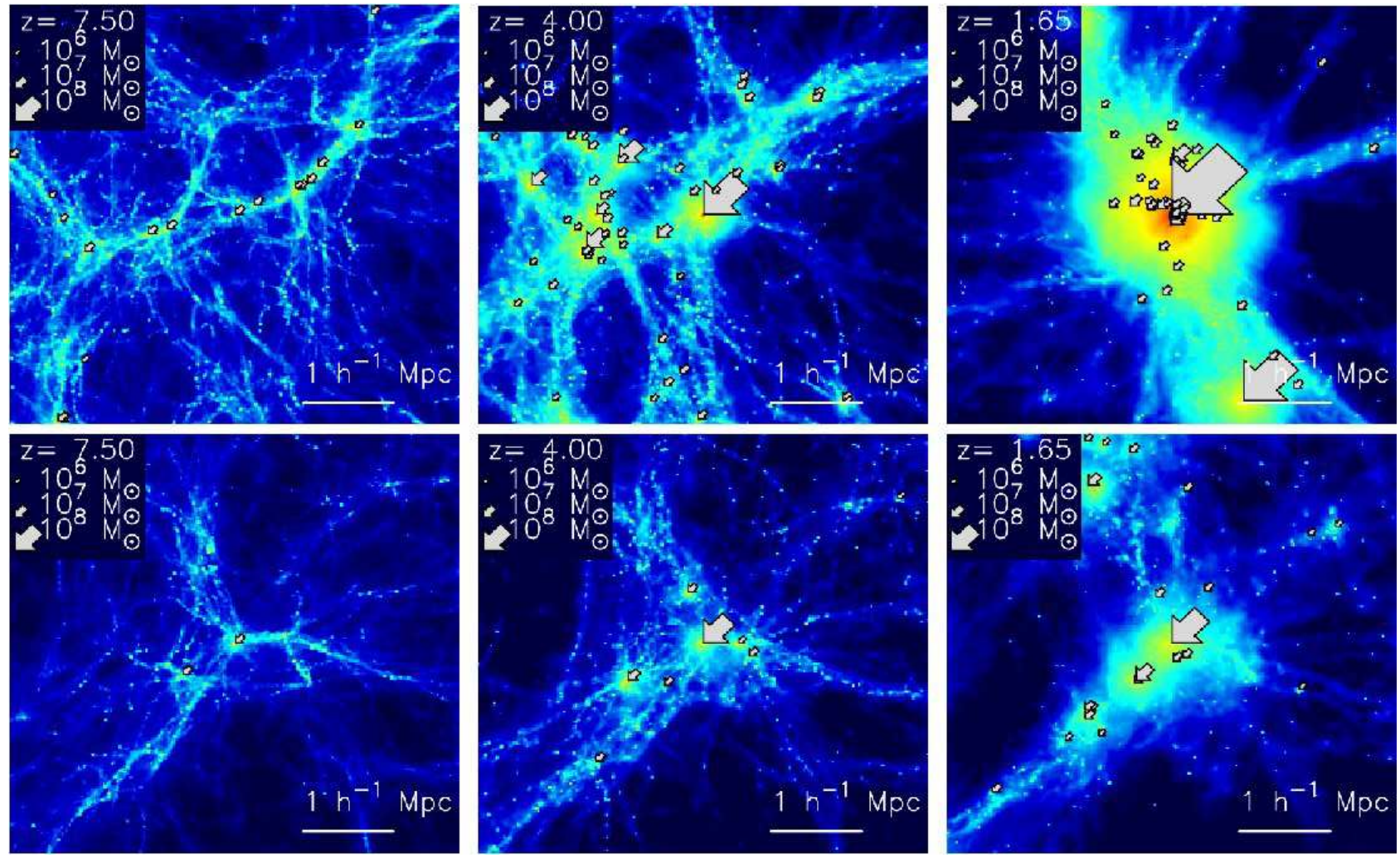

FIG. 14. - Time evolution of the environment around the host galaxy of the first supermassive massive black hole at $z=6$ (bottom panels), and of the most massive $\mathrm{BH}$ at $z=1$ (top panels) in the BHcosmo run. The location and masses of the supermassive BHs are marked by arrows of different size, as labeled. While the bottom system hosts the most massive black hole at high redshift, it does not end up hosting also the most massive black hole at the center of the largest galaxy at low redshift. Instead, the system shown in the top panels overtakes it in growth at intermediate redshifts, when it is formed in the highest density region in the simulation, which is a protocluster region.

including the most massive black hole at $z=6$, the most massive and second most massive at $z=1$, and finally one of the intermediate mass ones. Note that this is a small sub-sample of the several thousand black holes in the simulation. We focus here on the evolution of the most massive ones, and in particular on their early formation and the fate of their descendants. In Colberg et al. (2007), we study a complete sample of individual $\mathrm{BH}$ histories as a function of environments for the entire simulation.

The first interesting result shown in Figure 13 is that even in this small volume conditions exist that are conducive for exponential black hole growth, as required by the presence of quasars at $z \sim 6$ with the large observed masses. The evolution of the mass of the largest black hole in our simulation at early times is shown by the thick pink line. The corresponding accretion rate history (in the same color) shows a rapid succession of numerous phases of high accretion at the critical Eddington rate, between $5 \lesssim z \lesssim 7.5$. Below $z \sim 5$, the black hole is drastically more quiescent, except for a couple of sporadic Eddington phases at around $z \sim 2$. Interestingly, this first most massive black hole at $z \sim 6$ is not the most massive one at $z=1$. Instead, it is 'overtaken' in growth by another black hole shown with the blue thick line, which is the most massive black hole at the end of the simulation. The Eddington growth phases for this objects start at $z<6$ and extend all to way to $z \sim 3.5$. By $z \sim 4.5$, the black hole mass in this system catches up with that of the first massive black hole, and then keeps growing exponentially to $z \sim 4$.
In Figure 14, we show projected gas density maps of the host galaxies and the surrounding structures (at redshifts $z=$ 7.5, 4, and 1.6) for two of these black holes, the one that is most massive at $z=1$, and the one that is most massive at $z=6$. This provides good clues for the origin of the evolutionary difference between these two systems. Although the hosts of both black holes at $z=7.5$ are halos of similar mass, $\sim 10^{10} \mathrm{M}_{\odot}$, one of them lies at the intersection of three small filaments, leading to efficient gas cooling and a high star formation of $\sim 100 \mathrm{M}_{\odot} \mathrm{yr}^{-1}$. The host galaxy grows rapidly until $z=6$ but then its gas supply dwindles, and at $z \sim 4$ it is overtaken by the other black hole. This latter one lies on a very massive filament, which grows even more vigorously at this later time than its host galaxy (which has a SFR of $\sim 1000 \mathrm{M}_{\odot} \mathrm{yr}^{-1}$ at $z \sim 2$ ). In this way a large stellar spheroid is produced around the $\mathrm{BH}$, which eventually will end up at the center of a rich cluster of galaxies. Our results therefore show that a massive black hole that is found in the $\mathrm{cD}$ of large galaxy cluster at late times was not necessarily the most massive one at $z=6$, as it has been often assumed in the literature (e.g. Springel et al. 2005d). Since the growth history of black holes is intertwined with the non-linear processes of structure formation, individual growth histories of BHs can be complex and need not preserve the rank order in a group of BHs that start out with similar masses.

Further confirmation of this result from the BHCosmo simulation come from our E6 run (see Table 1), which includes the same physics and model parameters as the BHCosmo sim- 


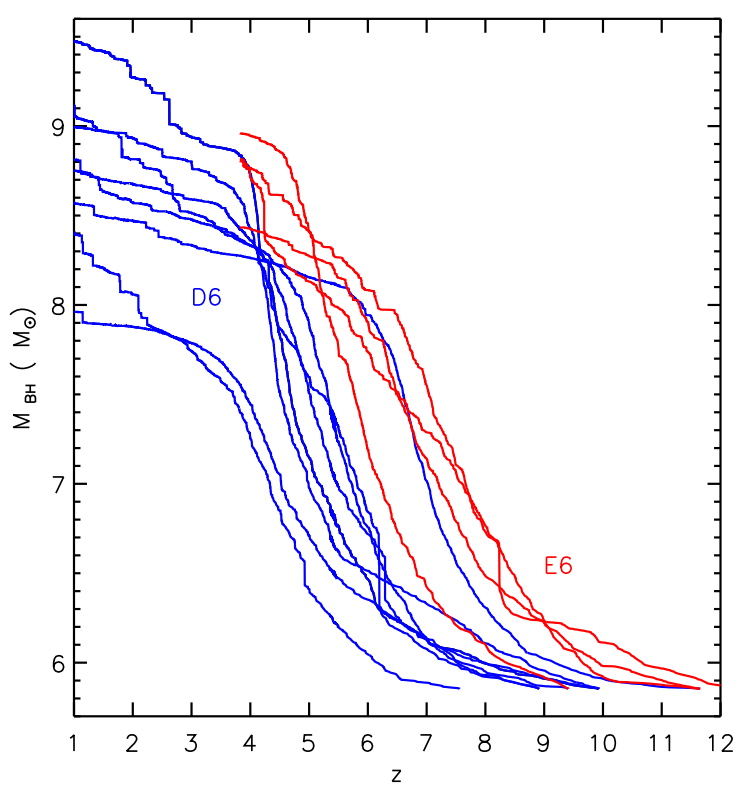

FIG. 15. - Comparison of individual black hole mass histories in the $\mathrm{BH}$ Cosmo run (blue lines, D6) and in the larger simulation volume of the E6 run (red lines). The growth of the first supermassive black holes at $z \sim 6-7$ is more widespread in the larger volume of the E6. The 'catch-up' of larger black holes that form later and in higher density regions (see text) can also be seen, and is comparable to the case shown in Fig. 13

ulation but samples rare halos better, thanks to its larger boxsize of $50 h^{-1} \mathrm{Mpc}$ on a side. In this larger volume, there are a handful of more examples of early exponential black hole growth between $8 \lesssim z \lesssim 6$, as shown by the red black hole mass growth curves in Figure 15. Consistent with our previous finding, there are again black holes that 'catch-up' in growth to the most massive black hole at some earlier time. We note that we have run this larger volume simulation with the specific purpose of checking the BHCosmo results for the first supermassive black holes by obtaining better statistics for large halos at $z \gtrsim 6$. For this reason, this simulations was not evolved beyond $z=4$.

\subsection{BH merger trees for the first and the most massive black hole}

Figures 16 and 17 show two example black hole merger trees, one for the most massive black hole at $z=1$, and one for the $z=1$ descendant of the first supermassive black hole at $z \sim 6$. All progenitor black holes that merge together to build up the final $\mathrm{BH}$ are included in the trees. The black hole masses along the tree are represented by different sizes of the circles, while the color encodes their accretion rate in units of the Eddington rate. In both figures, the top-left panel shows the full $\mathrm{BH}$ merger tree, whereas the top-right and bottom two panels split the tree according to accretion rate of the BHs.

Inspection of these two $\mathrm{BH}$ trees immediately reveals a complex and rich merger history for the most massive black holes at $z=1$ (Fig. 16), as opposed to the comparatively isolated evolution of the most massive object at $z=6$, which only features three major branches for its tree (Fig. 17). The large majority of the mass in the latter case is built up by early phases of critical gas accretion at $z \lesssim 6$. On the other hand, in Figure 16. Eddington accretion phases are spread out along many branches of the tree and cover a much larger range of redshifts. At redshifts $z<3$, the progenitors are preferentially accreting at low Eddington rates, so that the residual growth of the final black hole mass increasingly occurs from "dry" mergers with other black holes, consistent with our earlier results for the global black hole growth.

\section{SUMMARY AND DISCUSSION}

In this paper we have investigated the coupled formation and evolution of black holes and galaxies using state-of-theart cosmological hydrodynamic simulations of the $\Lambda \mathrm{CDM}$ model. For the first time, we have incorporated black hole growth and associated feedback from quasar activity self-consistently in cosmological hydrodynamic simulations. Our approach has been based on the methodology recently developed and tested in simulations of galaxy mergers (Di Matteo et al. 2005; Springel et al. 2005b). In this initial paper we have focused on investigating the model predictions for (i) the global history of black hole mass assembly and its relation to the history of star formation, from high redshift to the present, for (ii) the evolution of the $\mathrm{BH}$ mass function and accretion rate function and its connection to the observational "downsizing" phenomenon, for (iii) the correlations between black hole mass, velocity dispersion, and stellar mass of host galaxies, and finally, for (iv) the formation and fate of the first quasars and the properties of their hosts.

An important and highly encouraging first result has has been that the cosmological black hole mass density $\rho_{\mathrm{BH}}$ predicted by our high-resolution simulation reproduces the locally measured value and its extrapolation to higher redshift $(z<2.5)$, inferred from integrating the X-ray luminosity functions (Yu \& Tremaine 2002; Marconi et al. 2004; Shankar et al. 2004), or, more directly, the bolometric quasar luminosity function (Hopkins et al. 2007b). We predict a steep evolution of $\rho_{\mathrm{BH}}$ as a function of redshift, with a rise that is more rapid than that of the star formation rate. At $z>3$, $\rho_{\mathrm{BH}} / \rho_{*} \propto(1+z)^{-3}$ whereas there is at most weak evolution in the ratio $\rho_{\mathrm{BH}} / \rho_{*}$ at $z \lesssim 3$. Similarly, whilst the star formation rate density $\dot{\rho}_{*}$ broadly tracks the BHAR density $\dot{\rho}_{\mathrm{BH}}$ below $z \sim 2.5-3$, their ratio evolves steeply at higher redshifts, as $\dot{\rho}_{\mathrm{BH}} / \dot{\rho}_{*} \propto(1+z)^{-4}$. The SFR density peaks earlier than the BHAR density and exhibits a more gradual evolution with redshift compared to the BHAR. Only at redshifts below the peak of the BHAR, the BHAR and SFR rate densities start tracking each other.

Our results for the evolution of the BHAR density are broadly consistent with constraints obtained by Hopkins et al. (2007b) from a comprehensive analysis of a large sample of observational data sets, which allowed them to synthesize the evolution of the bolometric quasar luminosity density with redshift and to show that the luminosity density indeed peaks at $z \sim 2-3$, with a sharp drop towards higher redshifts.

We have shown that the growth of the black hole mass function shows signs of 'anti-hierarchical' behavior, or 'downsizing'. The high mass end of the $\mathrm{BH}$ mass function is established at comparatively high redshift and then significantly slows down in evolution below $z \sim 2$, where only the abundance of intermediate mass BHs is still growing appreciably. We have found more direct evidence for black hole "downsizing" by inspecting the distribution of accretion rates, in unis of the critical Eddington rate. The accretion rate function shifts from a narrow distribution dominated by high Eddington rates at high redshift to a broad distribution with a small fraction of Eddington accretion for low redshifts $(z<3)$. High mass black holes, forming in the high density peaks at high red- 


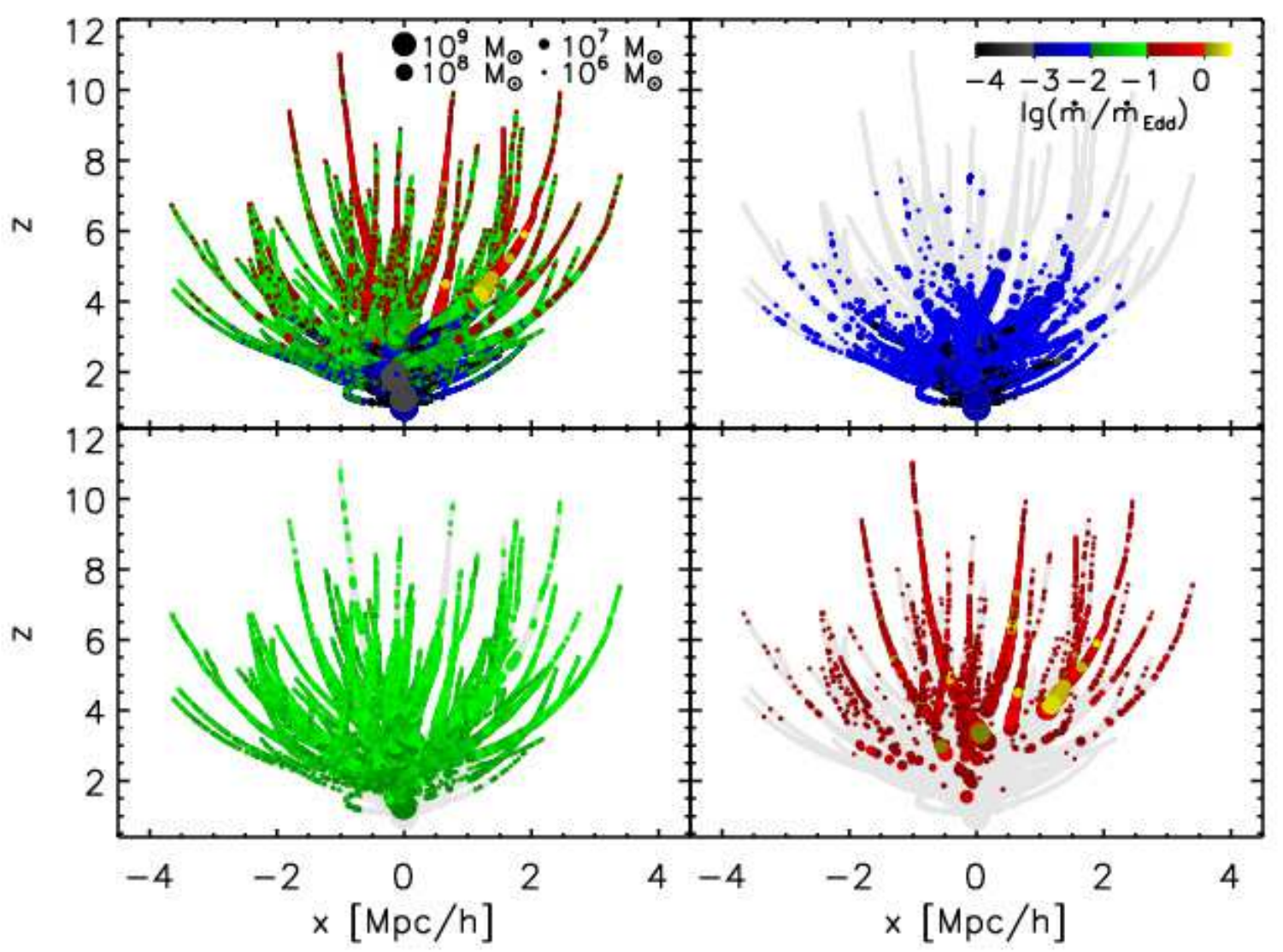

FIG. 16. - Black hole merger history trees shown as a function of redshift ( $y$-axis) and position (along the $x$-axis), for the most massive black hole at $z=1$ in the BHCosmo run. The full tree is shown in the top left panel, while the other three panels split up the tree according to accretion rate in units of Eddington, as indicated by the colors. The black hole mass is given by the size of the symbols, as labeled.

shift are built up rapidly by vigorous accretion. However, the effects of gas depletion and AGN feedback drive a strong decline in the accretion rate of massive black holes towards late times, while during these later times the peak of the accretion activity shifts to progressively smaller mass scales.

The BH masses of our simulated galaxies are strongly correlated with the stellar velocity dispersions and stellar masses of their host galaxies, and the correlations agree remarkably well with the local $M_{\mathrm{BH}}-\sigma$ and $M_{\mathrm{BH}}-M_{*}$ relationships, over a large dynamic range. We previously showed with simulations of isolated galaxy mergers (Di Matteo et al. 2005; Robertson et al. 2006b; Hopkins et al. 2007a) that our AGN feedback prescription leads to a self-regulated $\mathrm{BH}$ growth that can explain the $M_{\mathrm{BH}}-\sigma$ relation. It is highly reassuring that the same simulation model, with an unmodified feedback efficiency $\epsilon_{f}$ reproduces the observed $M_{\mathrm{BH}}-\sigma$ in full cosmological simulations as well. We emphasize that the free parameter $\epsilon_{f}$ sets the normalization of the obtained relationship, but the slope and scatter of the relation obtained from the simulations are not adjustable and a non-trivial consequence of the selfregulated $\mathrm{BH}$ growth.

Our simulation also suggests a weak evolution with redshift of the normalization of the $M_{\mathrm{BH}}-\sigma$ relation, as $M_{\mathrm{BH}} / \sigma \sim$ $(1+z)^{-0.2}$. However, we find that this evolutionary trend is sensitive to range of masses being probed. When we focus on the better resolved more massive systems, there appears to be some mild evolution in the slope of the $\mathrm{BH}$ scaling relations at high $\sigma$ and for high $M_{*}$. In particular, we find a trend of increasing $M_{*} / M_{\mathrm{BH}}$ with redshift, in agreement with recent direct estimates of the $\mathrm{BH}$ to host stellar mass ratio at high redshift by Peng et al. (2006). This trend is accompanied by an increase in the cold gas fraction in the host galaxy, and a smaller $R_{e}$ at a fixed stellar mass. These results are consistent with the suggestion that the $M_{\mathrm{BH}}-\sigma$ and $M_{\mathrm{BH}}-M_{*}$ relations are projections of a more basic correlation, the black hole fundamental plane (Hopkins et al. 2007a),$M_{\mathrm{BH}} \propto \sigma^{3} R_{e}^{0.5}$, and the interpretation that gas-rich systems at high redshift produce more dissipative mergers that lead to more concentrated $\mathrm{BH}$ hosts.

Our findings for the $\mathrm{BH}$ scaling relations appear fully consistent with a scenario where quasar activity is driven by galaxy mergers, as suggested by our simulations of isolated mergers (Di Matteo et al. 2005; Springel et al. 2005b; Robertson et al. 2006b; Hopkins et al. 2006a, 2007a). While gas is available for star formation over a large range of mass scales, it can only gets to central regions of galaxies in large amounts as a result of the inflows that accompany major mergers. At the same time, the mergers produce spheroids, which together with the growth-limiting quasar feedback establishes the $M_{\mathrm{BH}}-\sigma$ relationship. The detailed time evolution of the quasar activity in an individual galaxy depends on the angular momentum of the gas and the disk size and morphology. It is clear that the limited spatial resolution of our cosmological simulation is a severe limitation on how faithfully this can be tracked in our calculation, but because the final BH masses 


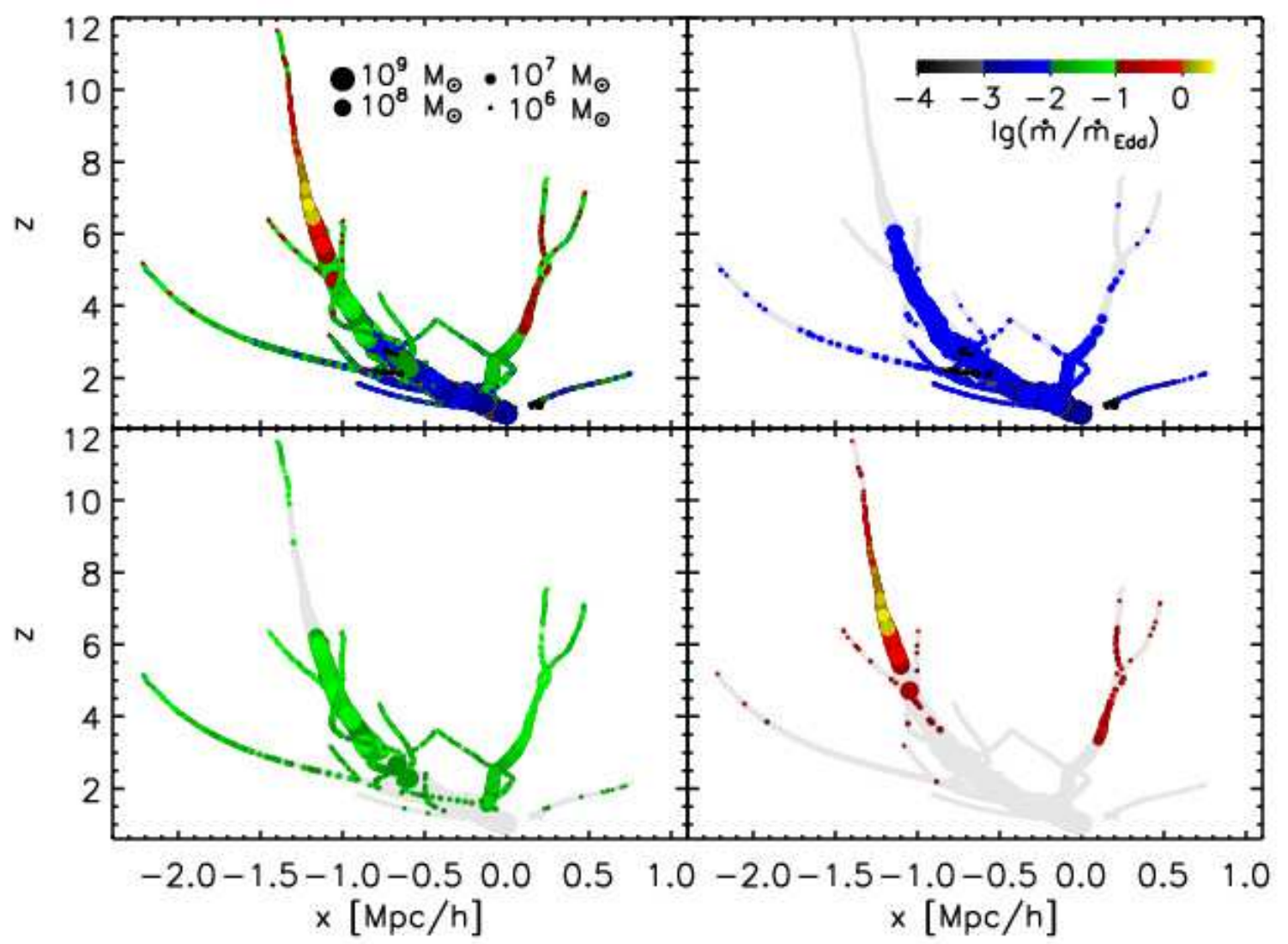

FIG. 17.- Black hole merger history tree shown as a function of redshift ( $y$-axis) and position ( $x$-axis) for the first high-redshift massive black hole that forms in the simulation. The full tree is shown in the top left panel, while the other three panels split up the tree according to accretion rate in units of Eddington, as indicated by the colors. The black hole mass is given by the size of the symbols, as labeled. The evolution of this system is qualitatively very different from the one shown in Fig. 16. Note that discreteness in the outputs for black holes that remain inactive cause the gap in the rightmost branch of this tree.

in merger remnants are robustly predicted by our simulation methodology even at coarse resolution this does not seriously affect the quantities we study here.

Our simulated volumes are too small to directly check if a population of supermassive black holes as massive the $z \sim 6$ Sloan quasars can form at high redshift. However, we do find that our simulation model produces black holes at early times that spend a large fraction of their time at high accretion rates close to their Eddington rates. We therefore predict the presence of massive black holes at high redshift. Using high-resolution simulations of multiple mergers that were constructed to represent a high redshift merging tree of one of the most massive protocluster regions in a $\left(1 h^{-1} \mathrm{Gpc}\right)^{3}$ volume, Li et al. (2007) have recently made the match with the Sloan quasar much more explicit. They were able to show that the gas-rich mergers expected in this rare overdensity can indeed grow a $\mathrm{BH}$ of mass $\sim 10^{9} \mathrm{M}_{\odot}$ early enough.

We have also found that the first supermassive black hole in our simulation forms in relative isolation, as a result of strong gas inflows and merging at the intersection of large-scale filaments. However, by following this system to lower redshift it turned out that it did not evolve into the most massive black hole today. Instead, it was overtaken in growth by a black hole that acquired most of its mass in major mergers in a high density region at lower redshift. Such a change in the relative rank of BHs as a function of time appears quite generic, as we explicitly checked with a simulation of larger volume.
This clearly complicates attempts to directly link high redshift progenitor systems to low redshift descendants. As we have demonstrated, the cosmological simulation methodology we introduced here provides however an excellent tool for studying the evolution of the cosmic $\mathrm{BH}$ evolution. In the present study, we focused only on the radiatively efficient accretion mode of AGN, which is associated with quasar activity. For following AGN activity also in clusters of galaxies, and hence down to $z=0$ in large volumes, we also need to account radio activity, which becomes important in very massive halos at low redshift. In Sijacki et al. (2007) we present an extension of our simulation methodology that accounts for this physics, and we discuss results obtained with this unified model for AGN feedback both for clusters of galaxies and cosmological boxes.

Our cosmological approach to black hole formation enables us to follow the fuel for black hole activity from its ultimate source, the early intergalactic medium, and so link the largescale structure and environments of black holes directly with their growth. As a result, countless different avenues of research are opened up and we plan to explore many in future work. For example, in Colberg et al. (2007) we use merger trees constructed for every black hole in the simulation to carry out a systematic study of their environments and histories and to determine the amount of black hole growth owing to mergers versus gas accretion; the latter should be testable with upcoming gravitational wave experiments. Future issues 
which will be addressed include the nature of AGN clustering and its evolution and its relation to the underlying dark matter clustering, predictions for quasar lifetimes and luminosity functions, and how feedback from AGN may manifest itself on large-scales and can be detected through the SunyaevZeldovich effect. With our new approach we can address the question of how the first black hole formed and grew we will be able to make predictions for the ionizing background owing to the first miniquasars, an important but currently uncer- tain ingredient in current models of reionization.

We thank Rupert Croft for many discussions and reading the manuscript. The simulations were performed at Carnegie Mellon University and the Pittsburgh Supercomputer Center (PSC). This work has been supported in part through NSF AST-0607819.

\section{REFERENCES}

Abel, T., Bryan, G. L., \& Norman, M. L. 2002, Science, 295, 93

Adelberger, K. L. \& Steidel, C. C. 2005, ApJ, 630, 50

Barnes, J. E. \& Hernquist, L. 1996, ApJ, 471, 115

Barnes, J. E. \& Hernquist, L. E. 1991, ApJ, 370, L65

Begelman, M. C., Blandford, R. D., \& Rees, M. J. 1980, Nature, 287, 307

Begelman, M. C., Volonteri, M., \& Rees, M. J. 2006, MNRAS, 370, 289

Bernardi, M., Sheth, R. K., Annis, J., Burles, S., Eisenstein, D. J., Finkbeiner,

D. P., Hogg, D. W., Lupton, R. H., Schlegel, D. J., SubbaRao, M., Bahcall,

N. A., Blakeslee, J. P., Brinkmann, J., Castander, F. J., Connolly, A. J.,

Csabai, I., Doi, M., Fukugita, M., Frieman, J., Heckman, T., Hennessy,

G. S., Ivezić, Ž., Knapp, G. R., Lamb, D. Q., McKay, T., Munn, J. A.,

Nichol, R., Okamura, S., Schneider, D. P., Thakar, A. R., \& York, D. G. 2003, AJ, 125, 1817

Bondi, H. 1952, MNRAS, 112, 195

Bondi, H. \& Hoyle, F. 1944, MNRAS, 104, 273

Bromm, V. \& Larson, R. B. 2004, ARA\&A, 42, 79

Bromm, V. \& Loeb, A. 2003, ApJ, 596, 34

Carilli, C. L., Walter, F., Bertoldi, F., Menten, K. M., Fan, X., Lewis, G. F. Strauss, M. A., Cox, P., Beelen, A., Omont, A., \& Mohan, N. 2004, AJ, 128,997

Carr, B. J. \& Rees, M. J. 1984, MNRAS, 206, 315

Cattaneo, A., Blaizot, J., Devriendt, J., \& Guiderdoni, B. 2005, MNRAS, 364 407

Cattaneo, A., Haehnelt, M. G., \& Rees, M. J. 1999, MNRAS, 308, 77

Ciotti, L. \& Ostriker, J. P. 1997, ApJ, 487, L105+

Colberg, J., Matteo, T. D., Springel, V., \& Hernquist, L. 2007, ArXiv Astrophysics e-prints

Croton, D. J., Springel, V., White, S. D. M., De Lucia, G., Frenk, C. S., Gao, L., Jenkins, A., Kauffmann, G., Navarro, J. F., \& Yoshida, N. 2006 MNRAS, 365, 11

De Lucia, G., Springel, V., White, S. D. M., Croton, D., \& Kauffmann, G. 2006, MNRAS, 366, 499

Di Matteo, T., Croft, R. A. C., Springel, V., \& Hernquist, L. 2003, ApJ, 593 , 56

Di Matteo, T., Springel, V., \& Hernquist, L. 2005, Nature, 433, 604

Escala, A., Larson, R. B., Coppi, P. S., \& Mardones, D. 2004, ApJ, 607, 765

Fabian, A. C. 1999, MNRAS, 308, L39

Fan, X., Strauss, M. A., Schneider, D. P., Becker, R. H., White, R. L., Haiman, Z., Gregg, M., Pentericci, L., Grebel, E. K., Narayanan, V. K., Loh, Y.S., Richards, G. T., Gunn, J. E., Lupton, R. H., Knapp, G. R., Ivezić, Ž. Brandt, W. N., Collinge, M., Hao, L., Harbeck, D., Prada, F., Schaye, J., Strateva, I., Zakamska, N., Anderson, S., Brinkmann, J., Bahcall, N. A., Lamb, D. Q., Okamura, S., Szalay, A., \& York, D. G. 2003, AJ, 125, 1649 Ferrarese, L. \& Merritt, D. 2000, ApJ, 539, L9

Gebhardt, K., Bender, R., Bower, G., Dressler, A., Faber, S. M., Filippenko,

A. V., Green, R., Grillmair, C., Ho, L. C., Kormendy, J., Lauer, T. R., Magorrian, J., Pinkney, J., Richstone, D., \& Tremaine, S. 2000, ApJ, 539, L13

Governato, F., Willman, B., Mayer, L., Brooks, A., Stinson, G., Valenzuela, O., Wadsley, J., \& Quinn, T. 2007, MNRAS, 374, 1479

Graham, A. W. \& Driver, S. P. 2006, ArXiv Astrophysics e-prints

Granato, G. L., De Zotti, G., Silva, L., Bressan, A., \& Danese, L. 2004, ApJ 600,580

Greenstein, J. L. \& Matthews, T. A. 1963, Nature, 197, 1041

Häring, N. \& Rix, H.-W. 2004, ApJ, 604, L89

Hasinger, G. Miyaji, T., \& Schmidt, R. 2005, MNRAS, in press

Hernquist, L. \& Springel, V. 2003, MNRAS, 341, 1253

Hopkins, P. F. \& Hernquist, L. 2006, ApJS, 166, 1

Hopkins, P. F., Hernquist, L., Cox, T. J., Di Matteo, T., Robertson, B., \& Springel, V. 2006a, ApJS, 163, 1

Hopkins, P. F., Hernquist, L., Cox, T. J., Robertson, B., \& Krause, E. 2007a, ArXiv Astrophysics e-prints

Hopkins, P. F., Hernquist, L., Cox, T. J., Robertson, B., Springel, V. Di Matteo, T., \& Springel, V. 2006b, ApJ, 639, 700
Hopkins, P. F., Hernquist, L., Martini, P., Cox, T. J., Robertson, B., Di Matteo, T., \& Springel, V. 2005, ApJ, 625, L71

Hopkins, P. F., Richards, G. T., \& Hernquist, L. 2007b, ApJ, 654, 731

Hoyle, F. \& Lyttleton, R. A. 1939, in Proceedings of the Cambridge Philisophical Society, 405

Jogee, S. 2004, in AGN Physics on All Scales

Katz, N., Weinberg, D. H., \& Hernquist, L. 1996, ApJS, 105, 19

Kauffmann, G. \& Haehnelt, M. 2000, MNRAS, 311, 576

Kennicutt, R. C. 1989, ApJ, 344, 685

-. 1998, ApJ, 498, 541

Kochanek, C. S., Pahre, M. A., Falco, E. E., Huchra, J. P., Mader, J., Jarrett, T. H., Chester, T., Cutri, R., \& Schneider, S. E. 2001, ApJ, 560, 566

Li, Y., Hernquist, L., Robertson, B., Cox, T. J., Hopkins, P. F., Springel, V., Gao, L., Di Matteo, T., Zentner, A. R., Jenkins, A., \& Yoshida, N. 2007 astro-ph/0608190

Lidz, A., Hopkins, P. F., Cox, T. J., Hernquist, L., \& Robertson, B. 2006, ApJ 641,41

Magorrian, J., Tremaine, S., Richstone, D., Bender, R., Bower, G., Dressler, A., Faber, S. M., Gebhardt, K., Green, R., Grillmair, C., Kormendy, J., \& Lauer, T. 1998, AJ, 115, 2285

Maiolino, R., Cox, P., Caselli, P., Beelen, A., Bertoldi, F., Carilli, C. L. Kaufman, M. J., Menten, K. M., Nagao, T., Omont, A., Weiß, A., Walmsley, C. M., \& Walter, F. 2005, A\&A, 440, L51

Makino, J. \& Funato, Y. 2004, ApJ, 602, 93

Malbon, R., Bough, C., Frank, C., \& Lacy, C. 2007, ArXiv Astrophysics eprints

Marconi, A., Risaliti, G., Gilli, R., Hunt, L. K., Maiolino, R., \& Salvati, M. 2004, MNRAS, 351, 169

Merloni, A. 2004, MNRAS, 353, 1035

Mihos, J. C. \& Hernquist, L. 1996, ApJ, 464, 641

Milosavljević, M. \& Merritt, D. 2003, ApJ, 596, 860

Monaghan, J. J. 1992, ARA\&A, 30, 543

Nagamine, K., Springel, V., Hernquist, L., \& Machacek, M. 2004, MNRAS, 350,385

Nakamura, O., Fukugita, M., Yasuda, N., Loveday, J., Brinkmann, J., Schneider, D. P., Shimasaku, K., \& SubbaRao, M. 2003, AJ, 125, 1682

Okamoto, T., Nemmen, R. S., \& Bower, R. G. 2007, ArXiv e-prints, 704

Peng, C. Y., Impey, C. D., Rix, H.-W., Kochanek, C. S., Keeton, C. R., Falco, E. E., Lehár, J., \& McLeod, B. A. 2006, ApJ, 649, 616

Robertson, B., Cox, T. J., Hernquist, L., Franx, M., Hopkins, P. F., Martini, P., \& Springel, V. 2006a, ApJ, 641, 21

Robertson, B., Hernquist, L., Cox, T. J., Di Matteo, T., Hopkins, P. F., Martini, P., \& Springel, V. 2006b, ApJ, 641, 90

Robertson, B., Yoshida, N., Springel, V., \& Hernquist, L. 2004, ApJ, 606, 32

Salucci, P., Szuszkiewicz, E., Monaco, P., \& Danese, L. 1999, MNRAS, 307, 637

Schmidt, M. 1963, Nature, 197, 1040

Shakura, N. I. \& Sunyaev, R. A. 1973, A\&A, 24, 337

Shankar, F., Salucci, P., Granato, G. L., De Zotti, G., \& Danese, L. 2004 MNRAS, 354, 1020

Sheth, R. K., Bernardi, M., Schechter, P. L., Burles, S., Eisenstein, D. J., Finkbeiner, D. P., Frieman, J., Lupton, R. H., Schlegel, D. J., Subbarao, M., Shimasaku, K., Bahcall, N. A., Brinkmann, J., \& Ivezić, Ž. 2003, ApJ, 594, 225

Shields, G. A., Gebhardt, K., Salviander, S., Wills, B. J., Xie, B., Brotherton, M. S., Yuan, J., \& Dietrich, M. 2003, ApJ, 583, 124

Shields, G. A., Menezes, K. L., Massart, C. A., \& Vanden Bout, P. 2006, ApJ, 641,683

Sijacki, D., Springel, V., Matteo, T. D., \& Hernquist, L. 2007, ArXiv Astrophysics e-prints

Silk, J. \& Rees, M. J. 1998, A\&A, 331, L1

Soltan, A. 1982, MNRAS, 200, 115 
Spergel, D. N., Bean, R., Doré, O., Nolta, M. R., Bennett, C. L., Dunkley, J., Hinshaw, G., Jarosik, N., Komatsu, E., Page, L., Peiris, H. V., Verde, L., Halpern, M., Hill, R. S., Kogut, A., Limon, M., Meyer, S. S., Odegard, N., Tucker, G. S., Weiland, J. L., Wollack, E., \& Wright, E. L. 2006, ArXiv Astrophysics e-prints

Spergel, D. N., Verde, L., Peiris, H. V., Komatsu, E., Nolta, M. R., Bennett, C. L., Halpern, M., Hinshaw, G., Jarosik, N., Kogut, A., Limon, M., Meyer, S. S., Page, L., Tucker, G. S., Weiland, J. L., Wollack, E., \& Wright, E. L. 2003, ApJS, 148, 175

Springel, V. 2005, MNRAS, 364, 1105

Springel, V., Di Matteo, T., \& Hernquist, L. 2005a, ApJ, 620, L79

-. 2005b, MNRAS, 361, 776

Springel, V. \& Hernquist, L. 2002, MNRAS, 333, 649

-. 2003a, MNRAS, 339, 289

-. 2003b, MNRAS, 339, 312

Springel, V., White, S. D. M., Jenkins, A., Frenk, C. S., Yoshida, N., Gao, L., Navarro, J., Thacker, R., Croton, D., Helly, J., Peacock, J. A., Cole, S.,

Thomas, P., Couchman, H., Evrard, A., Colberg, J., \& Pearce, F. 2005c, Nature, 435, 629

-. 2005d, Nature, 435, 629

Springel, V., Yoshida, N., \& White, S. D. M. 2001, New Astronomy, 6, 79
Tremaine, S., Gebhardt, K., Bender, R., Bower, G., Dressler, A., Faber, S. M., Filippenko, A. V., Green, R., Grillmair, C., Ho, L. C., Kormendy, J., Lauer, T. R., Magorrian, J., Pinkney, J., \& Richstone, D. 2002, ApJ, 574, 740

Trujillo, I., Förster Schreiber, N. M., Rudnick, G., Barden, M., Franx, M., Rix, H.-W., Caldwell, J. A. R., McIntosh, D. H., Toft, S., Häussler, B., Zirm, A., van Dokkum, P. G., Labbé, I., Moorwood, A., Röttgering, H., van der Wel, A., van der Werf, P., \& van Starkenburg, L. 2006, ApJ, 650, 18

Ueda, Y., Akiyama, M., Ohta, K., \& Miyaji, T. 2003, ApJ, 598, 886

Vestergaard, M. 2004, ApJ, 601, 676

Volonteri, M., Haardt, F., \& Madau, P. 2003, ApJ, 582, 559

Walter, F., Carilli, C., Bertoldi, F., Menten, K., Cox, P., Lo, K. Y., Fan, X., \& Strauss, M. A. 2004, ApJ, 615, L17

Willott, C. J., Percival, W. J., McLure, R. J., Crampton, D., Hutchings, J. B., Jarvis, M. J., Sawicki, M., \& Simard, L. 2005, ApJ, 626, 657

Woo, J.-H., Treu, T., Malkan, M. A., \& Blandford, R. D. 2006, ApJ, 645, 900 Wyithe, J. S. B. \& Loeb, A. 2003, ApJ, 595, 614

Yoshida, N., Omukai, K., Hernquist, L., \& Abel, T. 2006, ApJ, 652, 6

Yu, Q. \& Tremaine, S. 2002, MNRAS, 335, 965 